\title{
Inference on a New Lifetime Distribution under Progressive Type II Censoring for a Parallel-Series Structure
}

\author{
Atef F. Hashem $\mathbb{D}^{1}$ and Salem A. Alyami \\ ${ }^{1}$ Mathematics and Computer Science Department, Faculty of Science, Beni-Suef University, Beni-Suef, Egypt \\ ${ }^{2}$ Department of Mathematics and Statistics, Faculty of Science, Imam Mohammad Ibn Saud Islamic University (IMSIU), \\ Riyadh 13318, Saudi Arabia \\ Correspondence should be addressed to Atef F. Hashem; atef011264@science.bsu.edu.eg
}

Received 16 December 2020; Revised 13 January 2021; Accepted 27 January 2021; Published 19 February 2021

Academic Editor: Ahmed Mostafa Khalil

Copyright (C) 2021 Atef F. Hashem and Salem A. Alyami. This is an open access article distributed under the Creative Commons Attribution License, which permits unrestricted use, distribution, and reproduction in any medium, provided the original work is properly cited.

\begin{abstract}
A new lifetime distribution, called exponential doubly Poisson distribution, is proposed with decreasing, increasing, and upsidedown bathtub-shaped hazard rates. One of the reasons for introducing the new distribution is that it can describe the failure time of a system connected in the form of a parallel-series structure. Some properties of the proposed distribution are addressed. Four methods of estimation for the involved parameters are considered based on progressively type II censored data. These methods are maximum likelihood, moments, least squares, and weighted least squares estimations. Through an extensive numerical simulation, the performance of the estimation methods is compared based on the average of mean squared errors and the average of absolute relative biases of the estimates. Two real datasets are used to compare the proposed distribution with some other wellknown distributions. The comparison indicates that the proposed distribution is better than the other distributions to match the data provided.
\end{abstract}

\section{Introduction}

Sometimes, there is a need to generate a new distribution from a given distribution if the new distribution gives more flexibility to the data analysis in the sense of having a better fit, more variations of the hazard rate function (HRF), etc. Several methods can be implemented to generate new distributions. One of them is the compounding of distribution functions.

Suppose that $f(t \mid \lambda)$ and $p(\lambda)$ are two probability functions and $\lambda$ can take countable numbers $\lambda_{1}, \lambda_{2}, \ldots$ with masses $p_{j}$ at $\lambda_{j}, j=1,2, \ldots$ Then, the technique of compounding distributions takes the form

$$
f(t)=\sum_{j=1}^{\infty} p_{j} f_{j}(t),
$$

where $p_{j}=p\left(\lambda_{j}\right)$ and $f_{j}(t)=f\left(t \mid \lambda_{j}\right)$.

In lifetime testing experiments and reliability studies, exponential distribution (ED) is one of the most discussed distributions. However, for some practical applications where associated hazard rates are not constant, presenting monotone shapes, the exponential distribution does not provide an appropriate parametric fit. In the last decades, new classes of distributions have been proposed based on modifications of the ED to overcome such a problem.

Kuş [1] presented the exponential Poisson distribution (EPD), which has a decreasing hazard rate, as a lifetime distribution of a system, connected as a series structure, where the number of its components is a random variable (RV) subjecting to the truncated Poisson distribution (at zero) and the underlying lifetime distribution is exponential. Cancho et al. [2] obtained the Poisson exponential distribution (PED), which has an increasing hazard rate, as the lifetime of a system, connected as a parallel structure, in which the number of its components is a RV subjecting to the truncated Poisson distribution (at zero). A distribution named exponentiated exponential Poisson distribution (EEPD) for the series-parallel structure in which the number 
of components included in the series subsystem is random and that of the parallel subsystem is fixed was considered by Ristić and Nadarajah [3]. For more details about the compounding of distribution functions based on failures of a system, see, for example, Louzada et al. [4, 5], Mahmoudi and Sepahdar [6], Abdel-Hamid and Hashem [7, 8], and Nadarajah et al. [9].

In the implemented paper, we use the compounding of distributions concept to generate a new distribution based on the failures of a system connected as a parallel-series structure where the number of units in both the parallel and series subsystems is random. We name the proposed distribution exponential doubly Poisson distribution (EDPD) and then compare it with exponentiated Weibull Poisson distribution (EWPD), EEPD, EPD, PED, and ED. The EDPD accommodates decreasing, increasing, and upside-down bathtub-shaped hazard rates. These various shapes make it more versatile to suit multiple real data and are therefore regarded as one of the motivations of the EDPD. Some properties of the proposed distribution are studied. Four estimation methods for the included parameters, based on progressive type II censoring, are considered.

The EDPD's further motivation is focused on the failure of a parallel-series system. Suppose the system of units in a factory is designed according to the parallel-series structure. As shown in Figure 1, let $k$ denote the number of parallel subsystems functioning independently at a certain time, where $K$ is a RV (with a realization $k$ ) subjecting to a discrete distribution (geometric, power series, zero-truncated Poisson, etc.). Assume that each system of the parallel systems consists of $L_{j}$ (where $L_{j}, j=1, \ldots, k$, are RVs with realizations $l_{j}$ ) units, which are connected as a series structure. Their failure times, $X_{1 j}, \ldots, X_{L_{j}}, j=1, \ldots, k$, are independent and identically distributed (iid) RVs subjecting to a continuous lifetime distribution (Lomax, exponential, Weibull, Burr type XII, etc.). The success of the $j$-th system depends on the success of all the system units. In other words, the system fails just a one unit fails, i.e., $Z_{j}=\min \left(X_{1 j}, \ldots, X_{L_{j}}\right), j=1, \ldots, k$. Since the $k$ systems are connected as a parallel structure, then the factory's system can succeed when at least one of its parallel subsystems succeeds. In other words, it fails if all of the included subsystems fail. Let $T$ represent the failure time of the factory's system. Then

$$
T=\max _{1 \leq j \leq k} Z_{j}=\max _{1 \leq j \leq k} \min _{1 \leq i \leq l_{j}} X_{i j}
$$

where $X_{i j}, i=1, \ldots, l_{j}, j=1, \ldots, k$ are iid RVs.

The rest of the paper is structured as follows: the EDPD and some of its properties are presented in Section 2. Section 3 explores several methods of estimation. In Section 4 , a real example is given. Section 5 presents a simulation analysis accompanied by conclusions in Section 6 .

\section{Exponential Doubly Poisson Distribution}

In the current section, we obtain the EDPD by compounding Poisson and EDs based on failures of the parallel-series system. The probability density function (PDF), cumulative

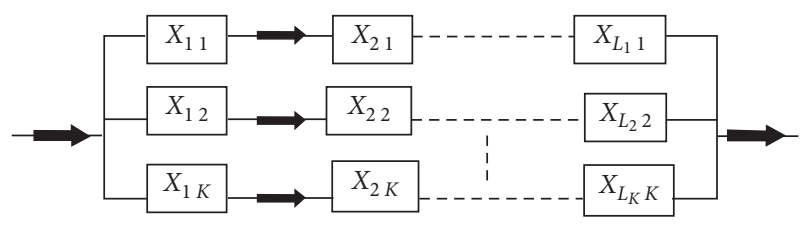

FIGURE 1: System with a parallel-series structure.

distribution function (CDF), survival function (SF), HRF, $r$-th moment, mean, variance, PDF of the $i$-th order statistic, Bonferroni curve, Lorenz curve, Rényi entropy, and Shannon's entropy of the EDPD are obtained and studied in detail.

The distribution of the RV T, presented in (2), is achieved in the following theorem.

Theorem 1. For $i=1, \ldots, l_{j}, j=1, \ldots, k$, suppose that $X_{i j}$, are iid $R V$ s with the $P D F f_{X}(x ; \theta)=\theta e^{-\theta x},(x, \theta>0)$ and the $C D F F_{X}(x ; \theta)=1-e^{-\theta x}$. Suppose also that $L_{j}$ and $K$ are two independent zero-truncated Poisson RVs with probability mass functions $P\left(L_{j}=l_{j} ; \sigma\right)=\left(\left(e^{-\sigma} \sigma^{l_{j}}\right) /\left(l_{j} !\left(1-e^{-\sigma}\right)\right)\right)$, $l_{j}=1,2, \ldots$ and $P(K=k ; \eta)=\left(\left(e^{-\eta} \eta^{k}\right) / k !\left(1-e^{-\eta}\right)\right), \quad k=$ $1,2, \ldots$, respectively. Then, the distribution of the $R V T$, presented in (2), has an EDPD with PDF and CDF given, respectively, by

$$
\begin{aligned}
& f(t) \equiv f(t ; \eta, \sigma, \theta)=\frac{\eta \sigma \theta}{\left(e^{\eta}-1\right)\left(e^{\sigma}-1\right)} \\
& \cdot e^{-\theta t+\sigma e^{-\theta t}} e^{\eta \Delta(t)}, \quad t>0,(\eta, \sigma, \theta>0), \\
& F(t) \equiv F(t ; \eta, \sigma, \theta)=\frac{e^{\eta \Delta(t)}-1}{e^{\eta}-1},
\end{aligned}
$$

where

$$
\Delta(t) \equiv \Delta(t ; \sigma, \theta)=\frac{e^{\sigma e^{-\theta t}}-e^{\sigma}}{1-e^{\sigma}} .
$$

Proof. Suppose that $Z_{j}=\min _{1 \leq i \leq l_{j}} X_{i j}, j=1, \ldots, k$.

The conditional density function of $Z_{j}$ given $L_{j}=l_{j}$ is then given by

$$
f\left(z_{j} \mid l_{j} ; \theta\right)=l_{j}\left[1-F_{X}\left(z_{j} ; \theta\right)\right]^{l_{j}-1} f_{X}\left(z_{j} ; \theta\right) .
$$

Using equation (1), the marginal PDF of $Z_{j}$ and its corresponding $\mathrm{CDF}$ are given, respectively, by

$$
\begin{aligned}
f_{Z}\left(z_{j} ; \sigma, \theta\right) & =\sum_{l_{j}=1}^{\infty} f\left(z_{j} \mid l_{j} ; \theta\right) P\left(L_{j}=l_{j} ; \sigma\right), \\
& =\frac{\sigma \theta e^{-\theta z_{j}}}{1-e^{-\sigma} e^{-\sigma\left(1-e^{-\theta z_{j}}\right)},} \\
F_{Z}\left(z_{j} ; \sigma, \theta\right) & =\frac{1-e^{-\sigma\left(1-e^{-\theta z_{j}}\right)}}{1-e^{-\sigma}} .
\end{aligned}
$$


Since $T=\max _{1 \leq j \leq k} \min _{1 \leq i \leq l_{i}} X_{i j}=\max _{1 \leq j \leq k} Z_{j}$, then the conditional density function of $T$ given $K=k$ is given by

$$
f(t \mid k ; \sigma, \theta)=k\left[F_{Z}(t ; \sigma, \theta)\right]^{k-1} f_{Z}(t ; \sigma, \theta) .
$$

Therefore, the marginal PDF of $T$ is given by

$$
\begin{aligned}
f(t ; \eta, \sigma, \theta) & =\sum_{k=1}^{\infty} f(t \mid k ; \sigma, \theta) P(K=k ; \eta), \\
& =\frac{\eta \sigma \theta}{\left(e^{\eta}-1\right)\left(e^{\sigma}-1\right)} e^{-\theta t+\sigma e^{-\theta t}} e^{\eta \Delta(t)},
\end{aligned}
$$

where $\Delta(t)$ is given by (5). Thus, the CDF of $T$ takes the form

$$
F(t ; \eta, \sigma, \theta)=\left(\frac{\left(e^{\eta \Delta(t)}-1\right)}{e^{\eta}-1}\right) \text {. }
$$

The SF and HRF of the EDPD with CDF (4) are given, respectively, by

$$
\begin{aligned}
& S(t)=\frac{e^{\eta}-e^{\eta \Delta(t)}}{e^{\eta}-1}, \quad t>0, \\
& h(t)=\frac{\eta \sigma \theta e^{-\theta t+\sigma e^{-\theta t}}}{\left(e^{\sigma}-1\right)\left(e^{\eta(1-\Delta(t))}-1\right)}, \quad t>0,
\end{aligned}
$$

where $\Delta(t)$ is given by (5).

Remark 1. The EDPD, with CDF (4), tends to the

(1) EPD, proposed by Kuş [1], if $\eta \longrightarrow 0^{+}$,

(2) PED, proposed by Cancho et al. [2], if $\sigma \longrightarrow 0^{+}$,

(3) $\mathrm{ED}$ if $\eta \longrightarrow 0^{+}$and $\sigma \longrightarrow 0^{+}$.

Theorem 2. A sufficient condition for PDF (3) to be decreasing (unimodal) is $\sigma+\left(\eta \sigma /\left(e^{-\sigma}-1\right)\right)+1>0(<0)$.

Proof. The derivation of $\log [f(t)]$ with respect to $t$ takes the form

$$
\frac{\mathrm{d} \log [f(t)]}{\mathrm{d} t}=-\theta e^{-\theta t} \Upsilon_{1}(t)
$$

where

$$
\Upsilon_{1}(t)=\sigma+\frac{\eta \sigma}{1-e^{\sigma}} e^{\sigma e^{-\theta t}}+e^{\theta t}
$$

The first derivative of the function $\Upsilon_{1}(t)$ takes the form

$$
\Upsilon_{1}^{\prime}(t)=\theta e^{\theta t}+\frac{\eta \sigma^{2} \theta}{e^{\sigma}-1} e^{-\theta t+\sigma e^{-\theta t}} .
$$

It is clear that $\Upsilon_{1}^{\prime}(t)>0, \forall t>0$. Therefore, the function $\Upsilon_{1}(t)$ is increasing.
Now, if $\Upsilon_{1}(0)=\sigma+\left(\eta \sigma /\left(e^{-\sigma}-1\right)\right)+1>0, \quad$ then $\Upsilon_{1}(t)>0, \forall t>0$ (since $\Upsilon_{1}(t)$ is increasing). Then $((\mathrm{d} \log [f(t)]) / \mathrm{d} t)<0$, and hence, the $\log [f(t)]$ is a decreasing function, which leads to $f(t)$ (PDF (3)) is also decreasing.

But, if $\Upsilon_{1}(0)=\sigma+\left(\eta \sigma /\left(e^{-\sigma}-1\right)\right)+1<0 \quad$ and $\Upsilon_{1}(\infty)=\infty$, then $\Upsilon_{1}(t)$ has a unique root $t_{0}$ and $\Upsilon_{1}(t)<0, \forall t<t_{0}$ and $\Upsilon_{1}(t)>0, \forall t>t_{0}$, and hence, the $\log [f(t)]$ is a unimodal function, which leads to $f(t)$ (PDF (3)) is also unimodal.

Theorem 3. HRF (12) of the EDPD is

(1) a decreasing (an increasing) function if $\eta<\left(\left(1-e^{-\sigma}\right) / 1+\sigma\right)\left(\eta>e^{\sigma}-1\right)$,

(2) an upside-down bathtub-shaped function if $\left(\left(1-e^{-\sigma}\right) /(1+\sigma)\right)<\eta<e^{\sigma}-1$.

Proof. Suppose that $\zeta(t)=\left(\left(-f^{\prime}(t)\right) / f(t)\right)$, where $f^{\prime}(t)=((\mathrm{d} f(t)) / \mathrm{d} t)$. Hence,

$$
\zeta(t)=\theta+\sigma \theta e^{-\theta t}+\frac{\eta \theta \sigma e^{-\theta t+\sigma e^{-\theta t}}}{1-e^{\sigma}},
$$

and its first derivative is $\zeta^{\prime}(t)=-\sigma \theta^{2} e^{-\theta t} \Upsilon_{2}(t)$, where

$$
\Upsilon_{2}(t)=1+\left(\left(\eta e^{\sigma e^{-\theta t}}\left(1+\sigma e^{-\theta t}\right)\right) /\left(1-e^{\sigma}\right)\right) .
$$

Its first derivative takes the form

$$
\Upsilon_{2}^{\prime}(t)=\frac{\eta \sigma \theta}{e^{\sigma}-1}\left(2+\sigma e^{-\theta t}\right) e^{-\theta t+\sigma e^{-\theta t}} .
$$

It is clear that $\Upsilon_{2}^{\prime}(t)>0, \forall t>0$. Therefore, the function $\Upsilon_{2}(t)$ is increasing.

Now, if $\quad \Upsilon_{2}(0)=1+((\eta(1+\sigma)) /$ $\left.\left(e^{-\sigma}-1\right)\right)>0, \Longleftrightarrow, \eta<\left(\left(1-e^{-\sigma}\right) /(1+\sigma)\right), \quad$ then $\Upsilon_{2}(t)>0, \forall t>0$. Thus, $\zeta^{\prime}(t)<0, \forall t>0$, and from Glaser's theorem (see Glaser [10]), HRF (12) is decreasing.

If $\quad \Upsilon_{2}(0)=1+\left((\eta(1+\sigma)) /\left(e^{-\sigma}-1\right)\right)<0 \Longleftrightarrow \eta>$ $\left(1-e^{-\sigma} / 1+\sigma\right) \quad$ and $\quad \Upsilon_{2}(\infty)=1+\left(\eta /\left(1-e^{\sigma}\right)\right)<0 \Longleftrightarrow$ $\eta>e^{\sigma}-1$, then $\Upsilon_{2}(t)<0, \forall t>0$. Thus, $\zeta^{\prime}(t)>0, \forall t>0$, and from Glaser's theorem, HRF (12) is increasing.

If $\Upsilon_{2}(0)=1+\left((\eta(1+\sigma)) /\left(e^{-\sigma}-1\right)\right)<0 \Longleftrightarrow \eta>\quad(1-$ $\left.\left.e^{-\sigma}\right) /(1+\sigma)\right) \quad$ and $\quad \Upsilon_{2}(\infty)=1+\left(\eta /\left(1-e^{\sigma}\right)\right)>$ $0 \Longleftrightarrow \eta<e^{\sigma}-1$, then $\Upsilon_{2}(t)$ has a unique root $t^{*}$ and $\Upsilon_{2}(t)<0, \forall t<t^{*}$, and $\Upsilon_{2}(t)>0, \forall t>t^{*}$. Thus, $\zeta^{\prime}(t)>0$, $\forall t<t^{*}$, and $\zeta^{\prime}(t)<0, \forall t>t^{*}$, and from Glaser's theorem, HRF (12) is upside-down bathtub.

For different values of $\eta, \sigma$, and $\theta, \operatorname{PDF}(3)$ and HRF (12) of the EDPD are plotted in Figure 2.

Theorem 4. Suppose that $T_{1}, \ldots, T_{n}$ is a random sample from the EDPD with CDF (4). Then, the CDF and its 

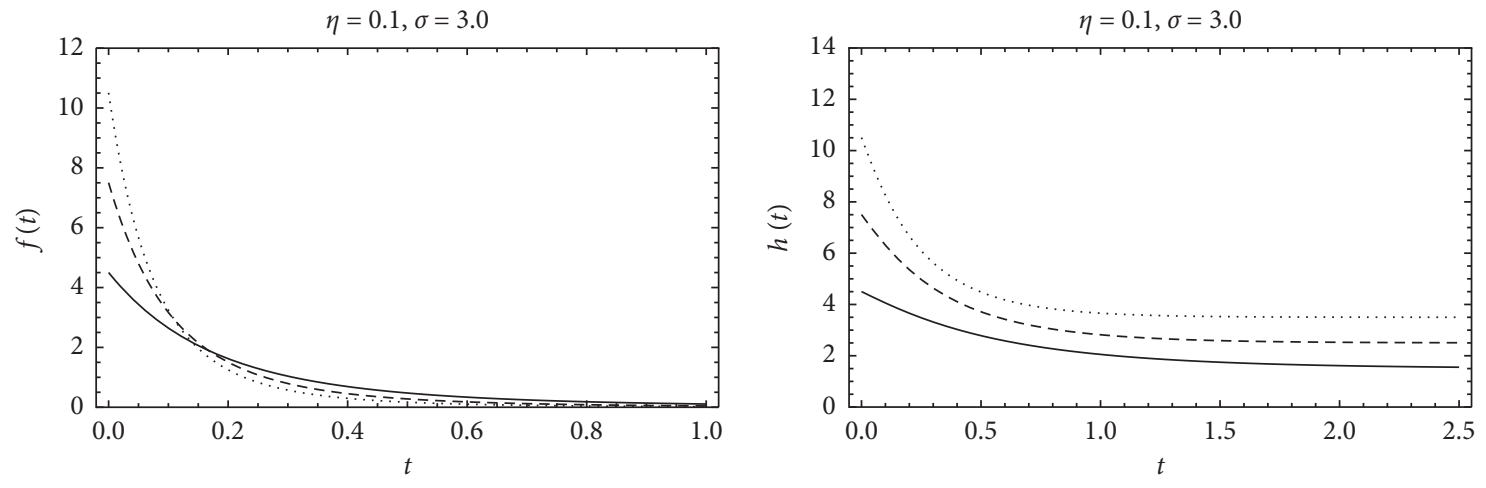
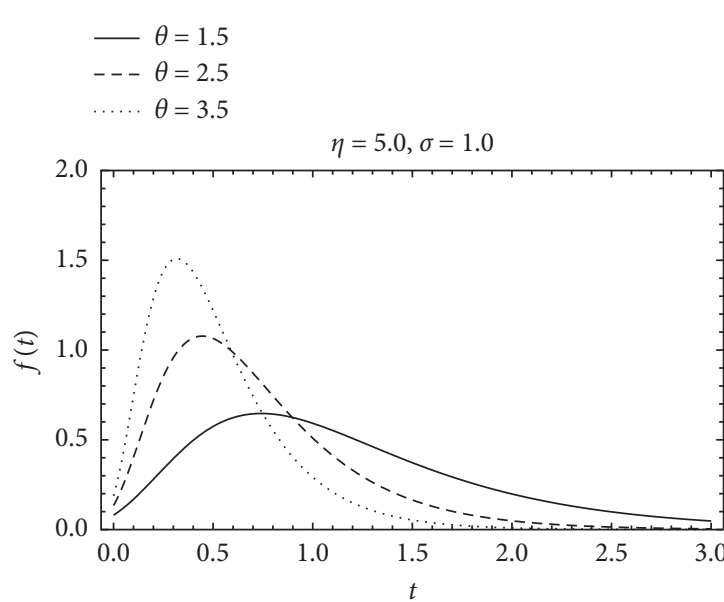

$-\theta=1.5$

$--\theta=2.5$

… $\theta=3.5$

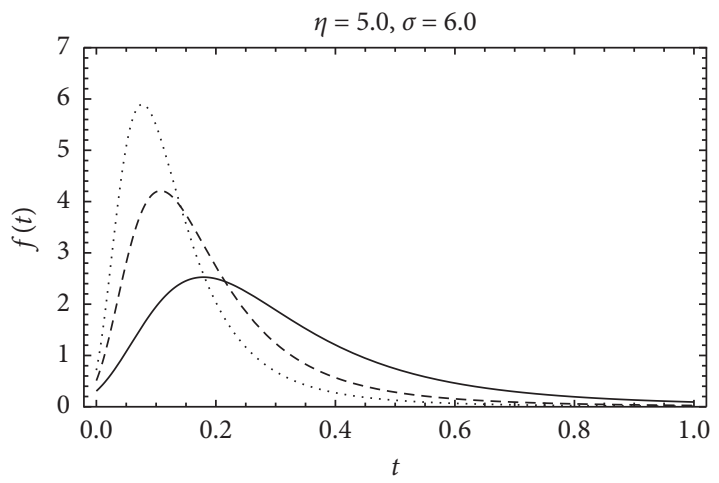

$-\theta=1.5$

$--\theta=2.5$

. $\theta=3.5$
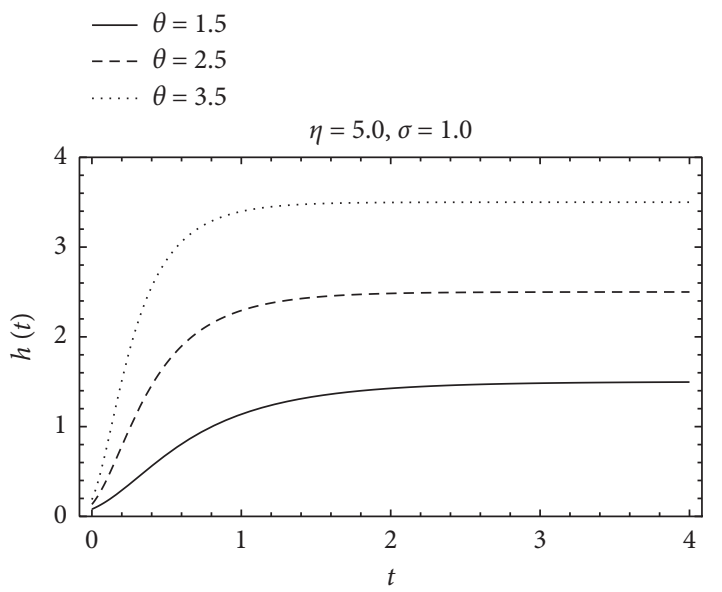

$\begin{aligned} \theta & =1.5 \\ --\theta & =2.5\end{aligned}$

.. $\theta=3.5$

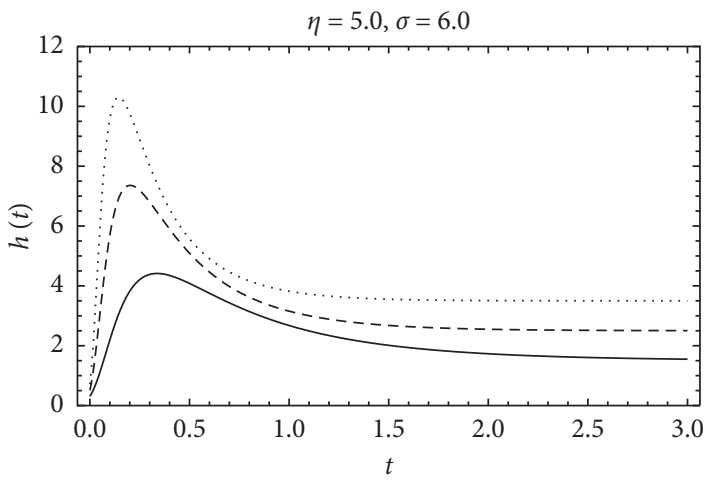

$\begin{aligned}-\theta & =1.5 \\ ---\theta & =2.5 \\ \ldots \theta \theta & =3.5\end{aligned}$

(a)

(b)

Figure 2: (a) The PDF and (b) HRF of the EDPD for different values of $\eta$, $\sigma$, and $\theta$. 
corresponding PDF of the $i$-th order statistic, say $T_{i: n}$, are given, respectively, by

$$
\begin{aligned}
& F_{i: n}(t)=\sum_{k_{3}=i}^{n} \sum_{k_{4}=0}^{n-k_{3}} \sum_{k_{5}=0}^{k_{3}+k_{4}}(-1)^{k_{4}+k_{5}}\left(\begin{array}{c}
n \\
k_{3}
\end{array}\right)\left(\begin{array}{c}
n-k_{3} \\
k_{4}
\end{array}\right)\left(\begin{array}{c}
k_{3}+k_{4} \\
k_{5}
\end{array}\right) \frac{e^{\eta k_{5} \Delta(t)}}{\left(1-e^{\eta}\right)^{k_{3}+k_{4}}}, \\
& f_{i: n}(t)=i\left(\begin{array}{c}
n \\
i
\end{array}\right) \frac{\eta \sigma \theta}{e^{\sigma}-1} e^{-\theta t+\sigma e^{-\theta t}} \sum_{k_{1}=0}^{n-i} \sum_{k_{2}=0}^{i+k_{1}-1}(-1)^{k_{1}+k_{2}-1}\left(\begin{array}{c}
n-i \\
k_{1}
\end{array}\right)\left(\begin{array}{c}
i+k_{1}-1 \\
k_{2}
\end{array}\right) \frac{e^{\eta\left(k_{2}+1\right) \Delta(t)}}{\left(1-e^{\eta}\right)^{i+k_{1}}}
\end{aligned}
$$

where $\Delta(t)$ is given by (5).

$$
f_{i: n}(t)=i\left(\begin{array}{c}
n \\
i
\end{array}\right) f(t)[F(t)]^{i-1}[1-F(t)]^{n-i} .
$$

Proof. As shown in Arnold et al. [11] and David and Nagaraja [12], the PDF $f_{i: n}(t)$ of the $i$-th order statistic can be written as follows:

If the $\operatorname{PDF} f(t)$ and $\operatorname{CDF} F(t)$ are given by equations (3) and (4), respectively, then

$$
\begin{aligned}
f_{i: n}(t) & =i\left(\begin{array}{c}
n \\
i
\end{array}\right) \sum_{k_{1}=0}^{n-i}(-1)^{k_{1}}\left(\begin{array}{c}
n-i \\
k_{1}
\end{array}\right) f(t)[F(t)]^{i+k_{1}-1} \\
& =i\left(\begin{array}{c}
n \\
i
\end{array}\right) \frac{\eta \sigma \theta}{e^{\sigma}-1} e^{-\theta t+\sigma e^{-\theta t}} \sum_{k_{1}=0}^{n-i} \sum_{k_{2}=0}^{i+k_{1}-1}(-1)^{k_{1}+k_{2}-1}\left(\begin{array}{c}
n-i \\
k_{1}
\end{array}\right)\left(\begin{array}{c}
i+k_{1}-1 \\
k_{2}
\end{array}\right) \frac{e^{\eta\left(k_{2}+1\right) \Delta(t)}}{\left(1-e^{\eta}\right)^{i+k_{1}}},
\end{aligned}
$$

where $\Delta(t)$ is given by (5).

The corresponding $\mathrm{CDF} F_{i: n}(t)$ is given by

$$
\begin{aligned}
F_{i: n}(t) & =\sum_{k_{3}=i}^{n}\left(\begin{array}{c}
n \\
k_{3}
\end{array}\right)[F(t)]^{k_{3}}[1-F(t)]^{n-k_{3}} \\
& =\sum_{k_{3}=i}^{n} \sum_{k_{4}=0}^{n-k_{3} \sum_{k_{3}+k_{4}}=0}(-1)^{k_{4}+k_{5}}\left(\begin{array}{c}
n \\
k_{3}
\end{array}\right)\left(\begin{array}{c}
n-k_{3} \\
k_{4}
\end{array}\right)\left(\begin{array}{c}
k_{3}+k_{4} \\
k_{5}
\end{array}\right) \frac{e^{\eta k_{5} \Delta(t)}}{\left(1-e^{\eta}\right)^{k_{3}+k_{4}}},
\end{aligned}
$$

where $\Delta(t)$ is given by (5).

$$
\begin{aligned}
b_{n}^{*}= & F^{-1}\left(\frac{1}{n}\right) \\
= & \frac{1}{\theta}\left\{-\log \left[\operatorname { l o g } \left(\left(1-e^{\sigma}\right) \log \left(-1+n+e^{\eta}\right)\right.\right.\right. \\
& \left.\left.\left.+(\eta+\log [n]) e^{\sigma}-\log [n]\right)-\log [\eta]\right]+\log (\sigma)\right\}, \\
a_{n}= & F^{-1}\left(1-\frac{1}{n}\right) \\
= & \frac{1}{\theta}\left\{-\log \left(\operatorname { l o g } \left[\eta e^{\sigma}+\log \left[1+n e^{\eta}-e^{\eta}\right]-\log [n]\right.\right.\right. \\
& \left.\left.-e^{\sigma} \log \left[1+n e^{\eta}-e^{\eta}\right]+e^{\sigma} \log [n]-\log [\eta]\right]+\log [\sigma]\right\}, \\
b_{n}= & \frac{1}{n f\left(a_{n}\right)} .
\end{aligned}
$$$$
\lim _{n \longrightarrow \infty} P\left(\frac{T_{n: n}-a_{n}}{b_{n}} \leq t\right)=e^{-e^{-t}}, \quad t \in \mathbb{R},
$$

where $a_{n}^{*}=0$, 
Proof. The proof can be easily shown by Theorem 8.3.1-Theorem 8.3.4 in Arnold et al. [11].

Theorem 6. Assume that $T_{1: n}<T_{2: n}<\cdots<T_{n: n}$ are order statistics from the EDPD. Then, for $i=1, \ldots, n$.

$$
\begin{gathered}
\lim _{n \longrightarrow \infty} P\left(\frac{T_{i: n}-a_{n}^{*}}{b_{n}^{*}} \leq t\right)=1-e^{-t} \sum_{j=0}^{i-1} \frac{t^{j}}{j !}, \quad t>0, \\
\lim _{n \longrightarrow \infty} P\left(\frac{T_{n-i+1: n}-a_{n}}{b_{n}} \leq t\right)=e^{-e^{-t}} \sum_{j=0}^{i-1} \frac{e^{-j t}}{j !}, \quad t \in \mathbb{R},
\end{gathered}
$$

where $a_{n}^{*}, b_{n}^{*}, a_{n}$, and $b_{n}$ are given in Theorem 5 .

Proof. Using equations (8.4.2) and (8.4.3) in Arnold et al. [11], the proof yields directly.

Theorem 7. If the RVT has the EDPD with PDF (3), then the $r$-th moment of $T$ is given by

$$
\mu_{r}=\sum_{\ell=0}^{N} \varpi_{\ell} \frac{2}{\left(1-y_{\ell}\right)^{2}}\left(\frac{1+y_{\ell}}{1-y_{\ell}}\right)^{r} f\left(\frac{1+y_{\ell}}{1-y_{\ell}}\right), \quad r=1,2, \ldots,
$$

where, as in Canuto et al. [13], $y_{\ell}$ and $\omega_{\ell}$ represent the zeros and corresponding Christoffel numbers of the Legendre-Gauss quadrature formula on the interval $(-1,1)$.

Proof. The $r$-th moment of the RV $T$ is given by

$$
\begin{aligned}
\mu_{r} & =\int_{0}^{\infty} t^{r} f(t) \mathrm{d} t \\
& =\int_{-1}^{1} \frac{2}{(1-y)^{2}}\left(\frac{1+y}{1-y}\right)^{r} f\left(\frac{1+y}{1-y}\right) \mathrm{d} y .
\end{aligned}
$$

We can approximate the previous integral, by using Legendre-Gauss quadrature formula as

$$
\mu_{r}=\sum_{\ell=0}^{N} \omega_{\ell} \frac{2}{\left(1-y_{\ell}\right)^{2}}\left(\frac{1+y_{\ell}}{1-y_{\ell}}\right)^{r} f\left(\frac{1+y_{\ell}}{1-y_{\ell}}\right),
$$

where

$$
\varpi_{\ell}=\frac{2}{\left(1-y_{\ell}^{2}\right)\left[L_{N+1}^{\prime}\left(y_{\ell}\right)\right]^{2}} \text {, and } L_{N+1}^{\prime}\left(y_{\ell}\right)=\frac{\mathrm{d} L_{N+1}(y)}{\mathrm{d} y} \text { at } y=y_{\ell} \text {, }
$$

and $L_{N}$ (.) is the Legendre polynomial of degree $N$.

Figure 3 plots the relationship between the mean and the degree $N$ of the Legendre polynomial in which we can observe the volume of $N$ required to obtain a satisfactory approximation to the true mean.

The mean, variance, skewness, and kurtosis of the EDPD are plotted in Figure 4. We observe the following:

(1) By increasing $\sigma$, the mean and variance decrease, but they increase by increasing $\eta$.

(2) By increasing $\eta$, the skewness and kurtosis decrease, but they increase-decrease by increasing $\sigma$.

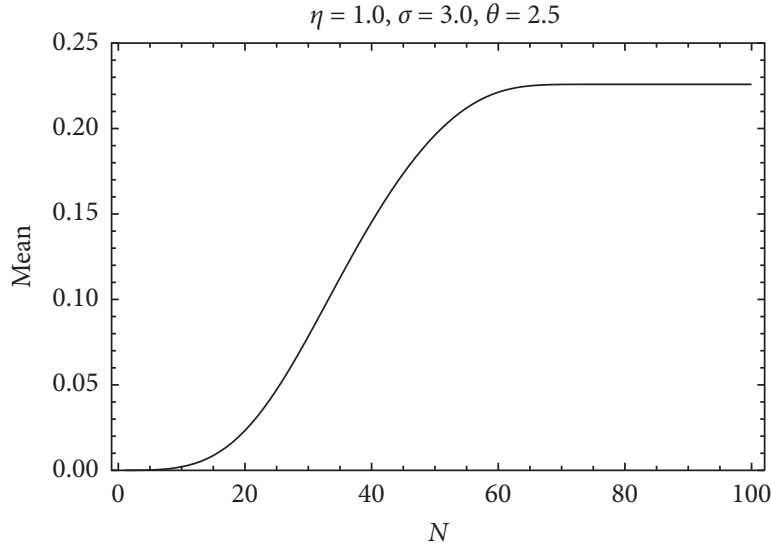

FIgURE 3: The relationship between the mean and the degree $N$ of Legendre polynomial.

The Bonferroni and Lorenz curves have applications in economics to study income and poverty, insurance, demography, reliability, and medicine. In the following theorem, we discuss the Bonferroni and Lorenz curves of the EDPD with PDF (3).

Theorem 8. Suppose that the RV T has the EDPD with PDF (3). Then, the Bonferroni curve $(B C(\xi))$ and Lorenz curve $(L C(\xi))$ are given, respectively, by

$$
\begin{aligned}
& B C(\xi)=\frac{q_{\xi}^{2}}{4 \xi \mu_{1}} \sum_{\ell=0}^{N} \varpi_{\ell}\left(y_{\ell}+1\right) f\left(\frac{q_{\xi}}{2}\left(y_{\ell}+1\right)\right), \\
& L C(\xi)=\frac{q_{\xi}^{2}}{4 \mu_{1}} \sum_{\ell=0}^{N} \varpi_{\ell}\left(y_{\ell}+1\right) f\left(\frac{q_{\xi}}{2}\left(y_{\ell}+1\right)\right),
\end{aligned}
$$

where $\omega_{\ell}$ is given by (29) and

$$
q_{\xi}=F^{-1}(\xi)=\frac{-1}{\theta} \log \left[\frac{1}{\sigma} \log \left(\frac{1-e^{\sigma}}{\eta} \log \left[\xi\left(e^{\eta}-1\right)+1\right]+e^{\sigma}\right)\right] .
$$

Proof. The Bonferroni curve of the EDPD is given by

$$
\begin{aligned}
B C(\xi) & =\frac{1}{\xi \mu_{1}} \int_{0}^{q_{\xi}} t f(t) \mathrm{d} t \\
& =\frac{q_{\xi}^{2}}{4 \xi \mu_{1}} \int_{-1}^{1}(y+1) f\left(\frac{q_{\xi}}{2}(y+1)\right) \mathrm{d} y \\
& =\frac{q_{\xi}^{2}}{4 \xi \mu_{1}} \sum_{\ell=0}^{N} \varpi_{\ell}\left(y_{\ell}+1\right) f\left(\frac{q_{\xi}}{2}\left(y_{\ell}+1\right)\right) .
\end{aligned}
$$

The Lorenz curve of the EDPD is given by

$$
\begin{aligned}
L C(\xi) & =\frac{1}{\mu_{1}} \int_{0}^{q_{\xi}} t f(t) \mathrm{d} t \\
& =\frac{q_{\xi}^{2}}{4 \mu_{1}} \sum_{\ell=0}^{N} \omega_{\ell}\left(y_{\ell}+1\right) f\left(\frac{q_{\xi}}{2}\left(y_{\ell}+1\right)\right) .
\end{aligned}
$$



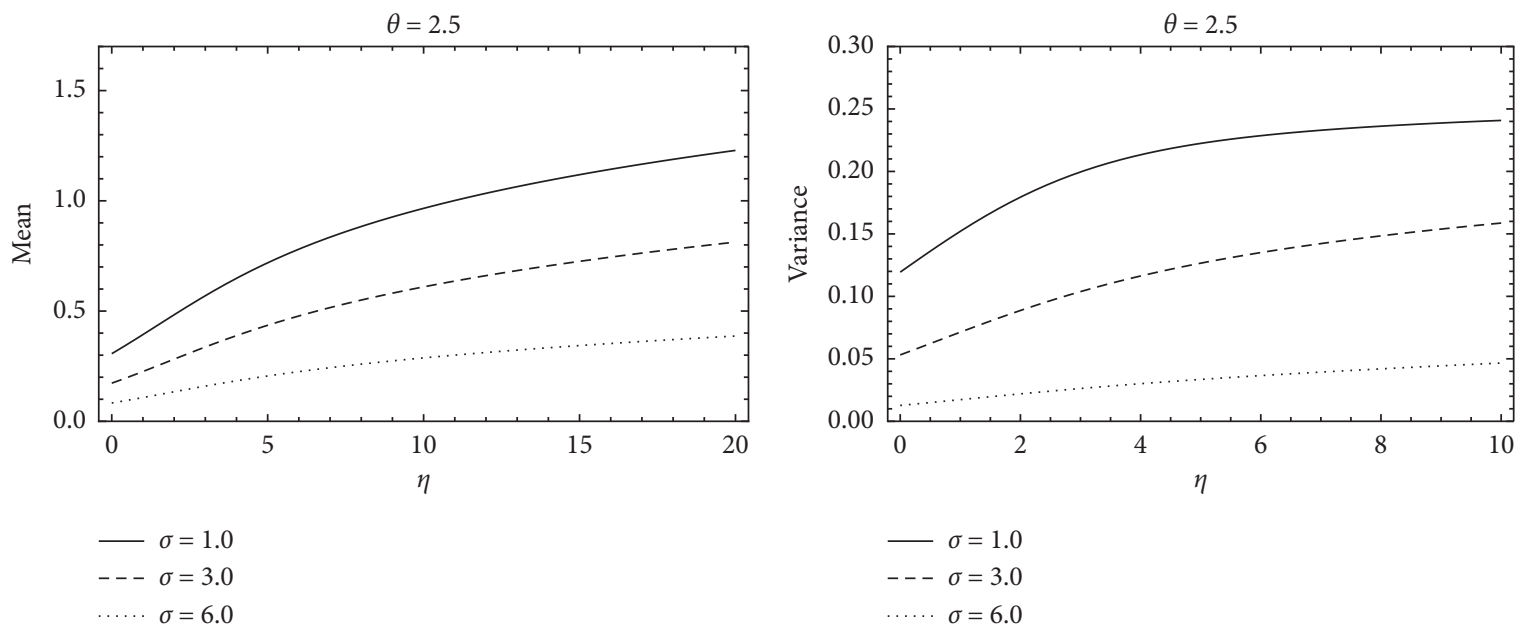

(a)

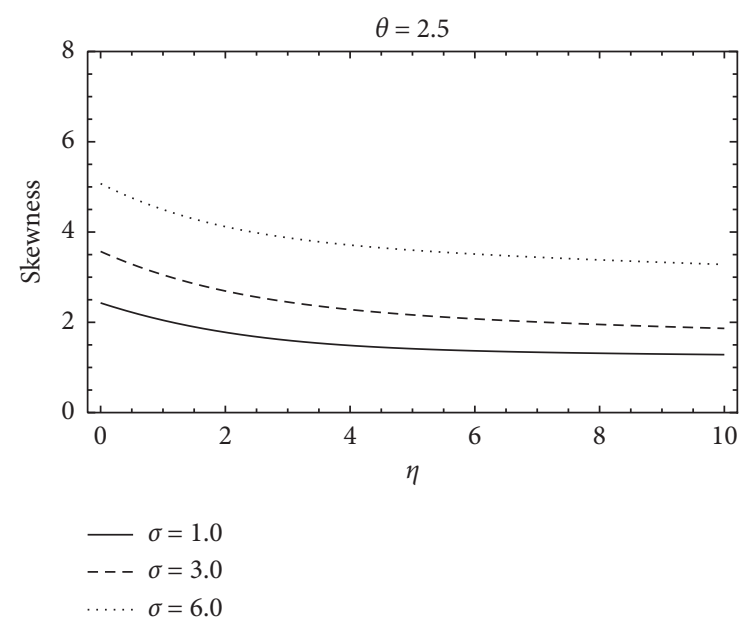

(b)

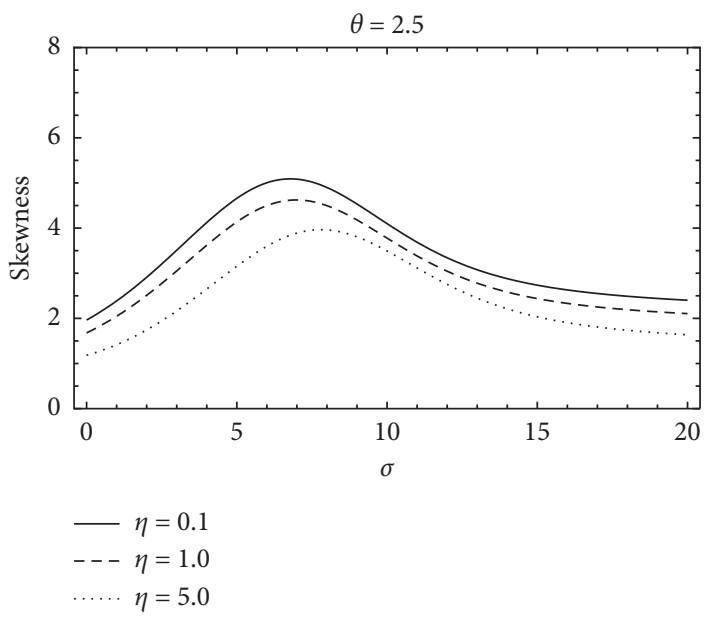

(c)

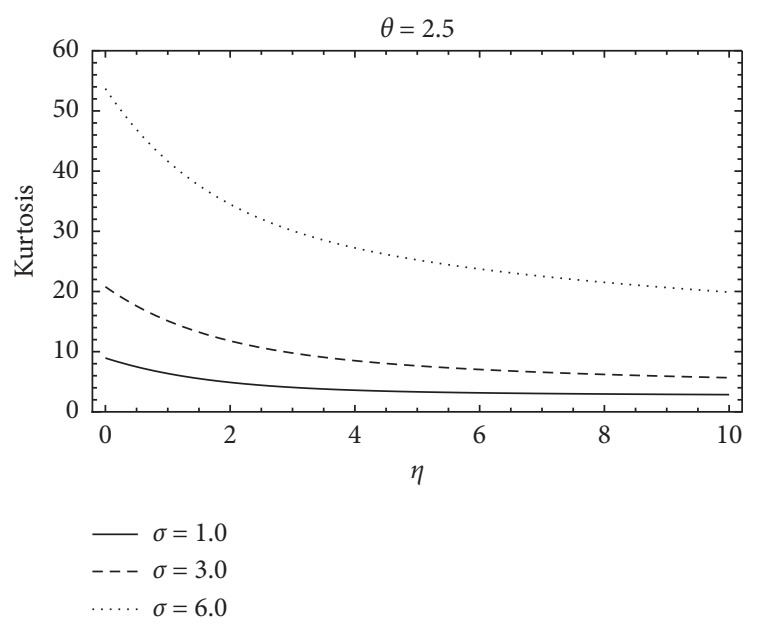

(d)

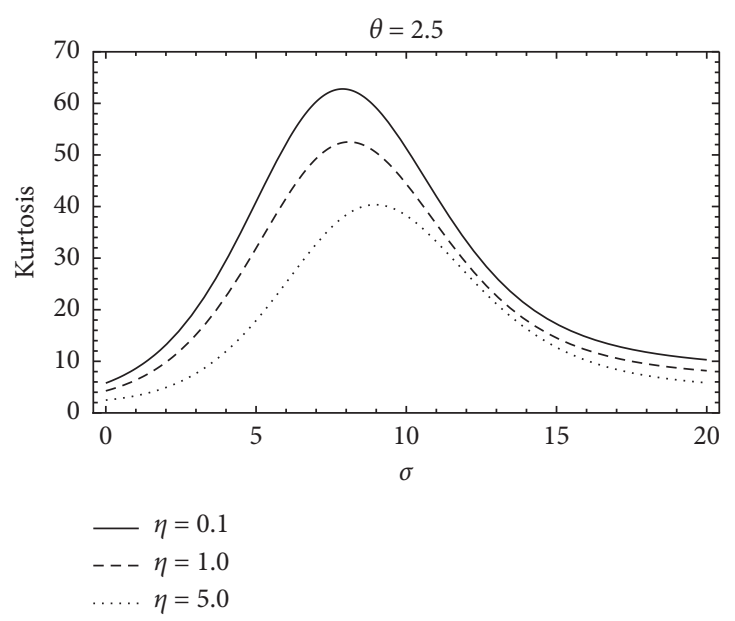

(e)

(f)

FIgURE 4: The mean, variance, skewness, and kurtosis of the EDPD.

The Bonferroni and Lorenz curves are plotted in Figure 5.

Entropy is applied to determine dynamical systems' randomness or uncertainty and is commonly applied in science and engineering. In the following theorem, we discuss two popular entropy measures that are the Rényi and Shannon's entropies (see Shannon [14] and Rényi [15]). 


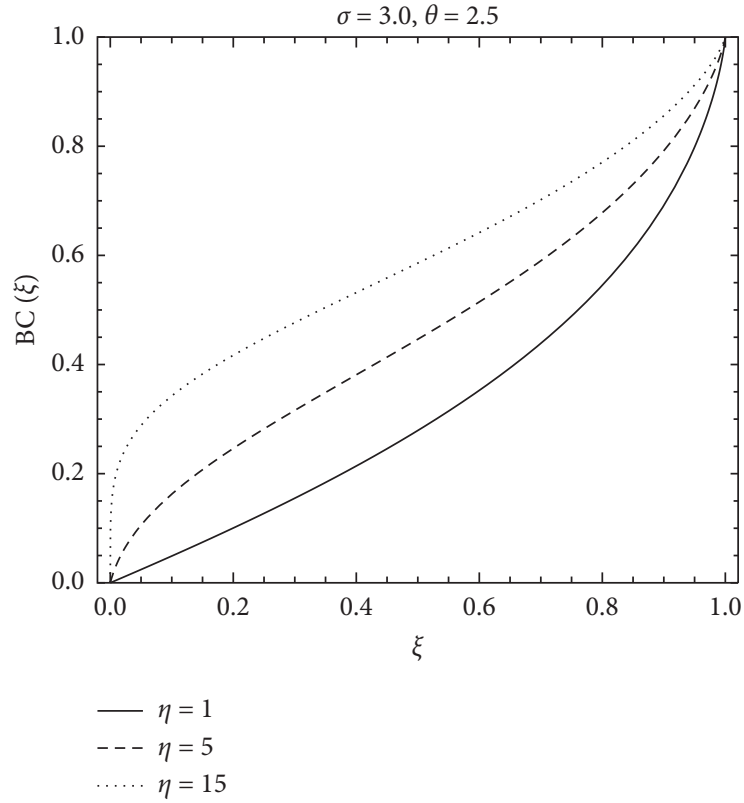

(a)

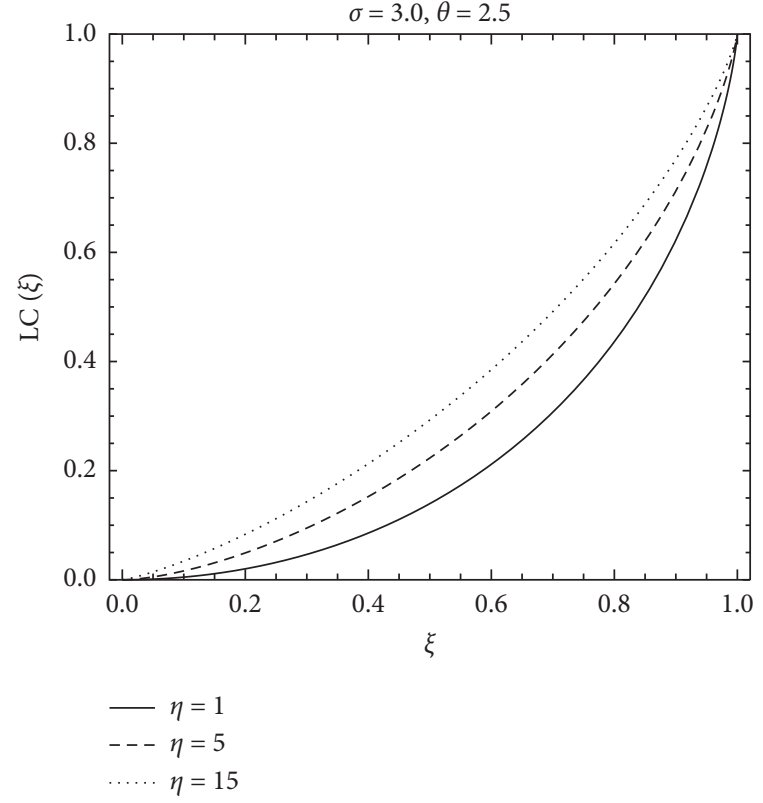

(b)

Figure 5: (a) The Bonferroni and (b) Lorenz curves of the EDPD.

Theorem 9. Let the RV T have the EDPD with PDF (3). Then, the Rényi and Shannon's entropies of $T$ are given, respectively, by

$$
\begin{aligned}
\mathbb{J}_{R}(\rho)= & \frac{1}{1-\rho}\left(\rho \log \left[\frac{\eta \sigma \theta}{\left(e^{\eta}-1\right)\left(e^{\sigma}-1\right)}\right]\right. \\
& \left.+\log \left[\sum_{\ell=0}^{N} \bigotimes_{\ell} \frac{2}{\left(1-y_{\ell}\right)^{2}} Q_{1}\left(\frac{1+y_{\ell}}{1-y_{\ell}}\right)\right]\right), \\
\mathbb{H}= & \log \left[\frac{\left(e^{\eta}-1\right)\left(e^{\sigma}-1\right)}{\eta \sigma \theta}\right]+\sum_{\ell=0}^{N}{\emptyset_{\ell}}_{\frac{2}{\left(1-y_{\ell}\right)^{2}} Q_{2}\left(\frac{1+y_{\ell}}{1-y_{\ell}}\right) f\left(\frac{1+y_{\ell}}{1-y_{\ell}}\right),}
\end{aligned}
$$

where $Q_{1}(t)=e^{\rho\left[-\theta t+\sigma e^{-\theta t}+\eta \Delta(t)\right]}, Q_{2}(t)=\theta t-\sigma e^{-\theta t}-\eta \Delta(t)$, and $\varpi_{\ell}$ is given by (29).

Proof. The Rényi entropy of $T$ is given by

$$
\begin{aligned}
\int_{0}^{\infty} f^{\rho}(t) \mathrm{d} t= & {\left[\frac{\eta \sigma \theta}{\left(e^{\eta}-1\right)\left(e^{\sigma}-1\right)}\right]^{\rho} \int_{0}^{\infty} Q_{1}(t) \mathrm{d} t } \\
= & {\left[\frac{\eta \sigma \theta}{\left(e^{\eta}-1\right)\left(e^{\sigma}-1\right)}\right]^{\rho} } \\
& \cdot \sum_{\ell=0}^{N} \Phi_{\ell} \frac{2}{\left(1-y_{\ell}\right)^{2}} Q_{1}\left(\frac{1+y_{\ell}}{1-y_{\ell}}\right) .
\end{aligned}
$$$$
\mathbb{J}_{R}(\rho)=\frac{1}{1-\rho} \log \left[\int_{0}^{\infty} f^{\rho}(t) \mathrm{d} t\right],
$$ 


$$
\begin{aligned}
\mathbb{J}_{R}(\rho)= & \frac{1}{1-\rho}\left(\rho \log \left[\frac{\eta \sigma \theta}{\left(e^{\eta}-1\right)\left(e^{\sigma}-1\right)}\right]\right. \\
& \left.+\log \left[\sum_{\ell=0}^{N} \Phi_{\ell} \frac{2}{\left(1-y_{\ell}\right)^{2}} Q_{1}\left(\frac{1+y_{\ell}}{1-y_{\ell}}\right)\right]\right) .
\end{aligned}
$$

Shannon's entropy of $T$ is given by

$$
\begin{aligned}
\mathbb{U}= & \mathrm{E}[-\log [f(t)]] \\
= & \log \left[\frac{\left(e^{\eta}-1\right)\left(e^{\sigma}-1\right)}{\eta \sigma \theta}\right]+\mathrm{E}\left[Q_{2}(t)\right] \\
= & \log \left[\frac{\left(e^{\eta}-1\right)\left(e^{\sigma}-1\right)}{\eta \sigma \theta}\right] \\
& +\sum_{\ell=0}^{N}{\Phi_{\ell}}_{\ell} \frac{2}{\left(1-y_{\ell}\right)^{2}} Q_{2}\left(\frac{1+y_{\ell}}{1-y_{\ell}}\right) f\left(\frac{1+y_{\ell}}{1-y_{\ell}}\right) .
\end{aligned}
$$

\section{Estimation Methods under Progressively Type II Censored Data}

Censoring may occur in medical or industrial applications when the experimenter is not able to obtain details of the total lifetime of each device or reduce the overall testing time and related cost. Types I and II are widely used censoring schemes (CS); see, for example, Mann et al. [16], Meeker and Escobar [17], and Lawless [18]. These two types of censoring cannot allow the experimenter to withdraw units from the life test at different times during the experiment. The experimenter can overcome this problem by using progressive type II censoring, which is a generalization of type II control. This type of censoring enables the experimenter to withdraw units from the life test at various times during the experiment (see Balakrishnan and Aggarwala [19]).

The progressive type II censoring can be performed as follows: suppose that the values of $m(<n)$ and $R_{1}, R_{2}, \ldots, R_{m}$ are assigned prior to the experiment. Once the first failure time occurs, $R_{1}$ functioning units are randomly withdrawn from the test. Also, once the second failure time occurs, $R_{2}$ surviving units are randomly withdrawn from the test. The test proceeds in the same way until occurring the $m$-th failure at which all the remaining functioning units $R_{m}=n-m-\sum_{j=1}^{m-1} R_{j}$ are withdrawn from the test, thereby terminating the life test. The data according to progressively type II censored samples are as follows: $\left(t_{1: m: n} ; R_{1}\right), \ldots$, $\left(t_{m: m: n} ; R_{m}\right)$ where $t_{1: m: n}<\cdots<t_{m: m: n}$ denote the $m$ ordered observed failure times, and $R_{1}, \ldots, R_{m}$ denote the number of units withdrawn from the experiment at failure times $t_{1: m: n}, \ldots, t_{m: m: n}$.

In what follows, based on progressive type II censoring, we consider four estimation methods to estimate the parameters $\eta, \sigma$, and $\theta$. The methods include the maximum likelihood (ML), moments (M), least squares (LS), and weighted least squares (WLS) estimations.

3.1. Maximum Likelihood Estimation Method. Let $\mathbf{t}=\left(t_{1}, \ldots, t_{m}\right), t_{i} \equiv t_{i: m: n}, i=1, \ldots, m$ be a progressively type II censored sample of size $m$ from the EDPD with PDF (3) and CDF (4). Then, the likelihood function is given by

$$
\mathbb{L}(\eta, \sigma, \theta ; \mathbf{t}) \propto \prod_{i=1}^{m} f\left(t_{i}\right)\left[1-F\left(t_{i}\right)\right]^{R_{i}}=\frac{\eta^{m} \sigma^{m} \theta^{m}}{\left(e^{\eta}-1\right)^{n}\left(e^{\sigma}-1\right)^{m}} e^{\sum_{i=1}^{m} \Psi_{i i}\left(t_{i}\right)},
$$

where

$$
\Psi_{1 i}\left(t_{i}\right)=-\theta t_{i}+\sigma e^{-\theta t_{i}}+\eta \Delta\left(t_{i}\right)+R_{i} \log \left[e^{\eta}-e^{\eta \Delta\left(t_{i}\right)}\right] .
$$

The logarithm of equation (39) is given by

$$
\begin{aligned}
E= & \log [\mathbb{L}(\eta, \sigma, \theta ; \mathbf{t})] \propto m \log [\eta \sigma \theta] \\
& -n \log \left[e^{\eta}-1\right]-m \log \left[e^{\sigma}-1\right]+\sum_{i=1}^{m} \Psi_{1 i}\left(t_{i}\right) .
\end{aligned}
$$

The likelihood equations for $\eta, \sigma$, and $\theta$ are given in Appendix A. No solution in a closed form could be obtained for the nonlinear likelihood equations to get the maximum likelihood estimates (MLEs) $\widehat{\eta}_{M L}, \widehat{\sigma}_{M L}$, and $\widehat{\theta}_{M L}$ of $\eta, \sigma$, and $\theta$. Therefore, the likelihood equations should be numerically manipulated to get the MLEs.

3.2. Moments Estimation Method. Let $T_{s: m: n}$ be the $s$-th progressively censored order statistic from an arbitrary continuous distribution with PDF $f(t)$. Then the $r$-th moment of $T_{s: m: n}$ is given by

$$
\mathrm{E}\left(T_{s: m: n}^{r}\right)=c \sum_{i=0}^{s-1} \frac{c_{i, s-1}\left(R_{1}+1, \ldots, R_{s-1}+1\right)}{R_{i}^{\prime \prime}} \bar{\mu}_{1: k_{i}^{\prime \prime}}^{r}
$$

where 


$$
\begin{aligned}
c & =n\left(n-R_{1}-1\right) \ldots\left(n-R_{1}-\cdots-R_{s-1}-s+1\right), \\
R_{i}^{\prime \prime} & =\left(R_{s}^{*}+1\right)+\sum_{j=s-i}^{s-1}\left(R_{j}+1\right), \\
R_{s}^{*} & =n-s-R_{1}-\cdots-R_{s-1}, \\
c_{i, l}\left(\mathbf{b}_{l}\right) & =\frac{(-1)^{i}}{\left[\prod_{j=1}^{i} \sum_{k=l-i+1}^{l-i+j} b_{l}\right]\left[\prod_{j=1}^{l-i} \sum_{k=j}^{l-i} b_{l}\right]}, \quad \mathbf{b}_{l}=\left(b_{1}, \ldots, b_{l}\right),
\end{aligned}
$$

and $\bar{\mu}_{1: R^{\prime \prime}}^{r}$ is the $r$-th moment of the usual smallest order statistic in a sample of size $R_{i}^{\prime \prime}$ from $f(t)$ (see Balakrishnan et al. [20]).

Let us discuss the moments estimates (MEs) based on a progressively type II censored data and consider the following system of equations:

$$
\sum_{s=1}^{m} \mathrm{E}\left(T_{s: m: n}^{r}\right)=\sum_{s=1}^{m} t_{s: m: n}^{r}, \quad r=1,2,3 .
$$

The MEs $\widehat{\eta}_{M}, \widehat{\sigma}_{M}$, and $\widehat{\theta}_{M}$ of $\eta, \sigma$, and $\theta$ can be obtained by solving numerically the above system of equations, where $\mathrm{E}\left(T_{s: m: n}^{r}\right)$ can be supplied by (42).

3.3. Least Squares and Weighted Least Squares Estimation Methods. The LS and WLS methods were introduced by Swain et al. [21] to estimate the beta distribution parameters. They can be performed as follows: let $T_{1: m: n}, \ldots, T_{m: m: n}$ be the ordered progressively type II censored sample of size $m$ from the EDPD. As in Aggarwala and Balakrishnan [22], the expectation and variance of the empirical CDF are given, respectively, by

$$
\begin{aligned}
& E\left[\widehat{F}\left(t_{i}\right)\right]=1-\prod_{j=m-i+1}^{m} \frac{\mathscr{W}_{j}}{1+\mathscr{W}_{j}}, \quad i=1, \ldots, m, \\
& V\left[\widehat{F}\left(t_{i}\right)\right]=\left(\prod_{j=m-i+1}^{m} \frac{\mathscr{W}_{j}}{1+\mathscr{W}_{j}}\right)\left(\prod_{j=m-i+1}^{m}\left[\frac{\mathscr{W}_{j}}{1+\mathscr{W}_{j}}+\frac{1}{\left(\mathscr{W}_{j}+1\right)\left(\mathscr{W}_{j}+2\right)}\right]-\prod_{j=m-i+1}^{m} \frac{\mathscr{W}_{j}}{1+\mathscr{W}_{j}}\right),
\end{aligned}
$$

where

$$
\mathscr{W}_{j}=j+\sum_{s=m-j+1}^{m} R_{s}, \quad j=1, \ldots, m .
$$

The LS estimates $\left(\widehat{\eta}_{L} \widehat{\sigma}_{L}, \widehat{\theta}_{L}\right)$ and WLS estimates $\left(\widehat{\eta}_{W} \widehat{\sigma}_{W}, \widehat{\theta}_{W}\right)$ of $(\eta, \sigma, \theta)$ could be obtained by minimizing the next two quantities, respectively, with respect to $(\eta, \sigma, \theta)$.

$$
\begin{aligned}
& \Phi_{1} \equiv \Phi_{1}(\boldsymbol{\lambda} ; \mathbf{t})=\sum_{i=1}^{m}\left(F\left(t_{i}\right)-E\left[\widehat{F}\left(t_{i}\right)\right]\right)^{2}, \\
& \Phi_{2} \equiv \Phi_{2}(\boldsymbol{\lambda} ; \mathbf{t})=\sum_{i=1}^{m} \frac{1}{V\left[\widehat{F}\left(t_{i}\right)\right]}\left(F\left(t_{i}\right)-E\left[\widehat{F}\left(t_{i}\right)\right]\right)^{2},
\end{aligned}
$$

where $\lambda=\left(\lambda_{1}=\eta, \lambda_{2}=\sigma, \lambda_{3}=\theta\right)$.

It is possible to minimize the last two quantities by solving the equations $\left(\partial \Phi_{1} / \partial \lambda_{r}\right)=0$ and $\left(\partial \Phi_{2} / \partial \lambda_{r}\right)=0$ with respect to $\lambda_{r}, r=1,2,3$.

\section{Real Data Example}

Two real datasets are proposed, in this section, to fit and compare the EDPD, EEPD (Ristić and Nadarajah [3]), EWPD (Mahmoudi and Sepahdar [6]), PED (Cancho et al. [2]), EPD (Kuş [1]), and traditional ED.

(i) The first data:

The first dataset presents the amount of annual rainfall (in inches) recorded at Los Angeles Civic
Center from 1922 to 2006 . The dataset consists of 85 observations as follows:

$13.19,37.96,9.25,16.44,4.35,17.94,11.57,9.09$, $31.01,12.40,12.46,24.35,8.11,27.36,21.0,11.99$, $7.35,8.08,12.48,7.66,17.86,12.82,10.43,31.25$, $10.71,8.98,26.98,19.67,33.44,12.31,7.22,14.35$, $14.92,21.26,7.17,12.32,7.77,27.47,16.58,22.41$, $20.44,13.69,7.93,8.38,18.79,4.85,8.18,5.58,21.13$, $9.54,16.0,11.94,11.99,9.46,26.21,8.21,9.94,7.99$, $7.22,12.66,11.65,11.59,19.17,18.17,11.18,32.76$, $19.21,13.07,23.43,22.41,12.07,21.66,14.55,11.88$, $16.95,12.53,11.52,12.66,9.77,17.7,17.56,7.94,6.67$, 9.59, and 19.66 .

Madi and Raqab [23] and Raqab et al. [24] used the amount of annual rainfall (in inches) recorded at Los Angeles Civic Center as a real dataset.

(ii) The second data:

The second dataset consists of the strengths of $1.5 \mathrm{~cm}$ glass fibres, measured at the National Physical Laboratory, England (see Smith and Naylor [25]). The dataset consists of 63 observations as follows:

$0.55,0.74,0.77,0.81,0.84,1.24,0.93,1.04,1.11,1.13$, $1.30,1.25,1.27,1.28,1.29,1.48,1.36,1.39,1.42,1.48$, $1.51,1.49,1.49,1.50,1.50,1.55,1.52,1.53,1.54,1.55$, $1.61,1.58,1.59,1.60,1.61,1.63,1.61,1.61,1.62,1.62$, $1.67,1.64,1.66,1.66,1.66,1.70,1.68,1.68,1.69,1.70$, $1.78,1.73,1.76,1.76,1.77,1.89,1.81,1.82,1.84,1.84$, $2.00,2.01$, and 2.24 . 
Some descriptive statistics for the two given datasets are presented in Table 1.

Based on the above two datasets, the Kolmogorov-Smirnov (K-S) statistic and its corresponding $p$ value are used to check the validity of the considered distributions as shown in Table 2. A comparison among the EDPD, EWPD, EEPD, EPD, PED, and the ED using some criteria such as the Akaike information criterion (AIC), consistent AIC (CAIC), and Bayesian information criterion (BIC) is also shown in Table 2, where

$$
\begin{aligned}
\mathrm{AIC} & =2 r-2 E(\widehat{\beta}), \\
\mathrm{CAIC} & =\frac{2 r m}{m-r-1}-2 E(\widehat{\beta}), \\
\mathrm{BIC} & =r \log [m]-2 E(\widehat{\beta}),
\end{aligned}
$$

where $E(\widehat{\beta})$ stands for the log-likelihood function calculated at the MLE $\widehat{\beta}$ of $\beta, r$ is the number of parameters, and $m$ is the sample size.

Based on the values of K-S statistic and its corresponding $p$ value presented in Table 2 , it can be noticed that the EDPD has the smallest (largest) K-S ( $p$ ) values than those for the EWPD, EEPD, EPD, PED, and ED. Therefore, the EDPD fits the considered data better than the five mentioned distributions. Since the EDPD has the smallest values of AIC, CAIC, and BIC, then this is considered another indicator of the superiority of the EDPD. The comparison is also shown graphically by plotting the empirical CDF against the CDF of EDPD, EWPD, EEPD, EPD, PED, and ED (see Figure 6).

It can be easily shown that the values of $\eta, \sigma$, and $\theta$ presented in Table 2 maximize likelihood function (19). We generate two progressively type II censored samples from the above two datasets as follows:

(i) The First Progressively Type II Censored Sample Suppose $m=50$ and then apply the following to the first dataset

$$
\begin{aligned}
& R_{i}=1, \quad i=1, \ldots, 35, \\
& R_{i}=0, \quad i=36, \ldots, 50 .
\end{aligned}
$$

Then the first progressively type II censored sample is $4.35,4.85,5.58,6.67,7.17,7.22,7.22,7.35,7.66$, 7.77, 7.93, 7.94, 7.99, 8.08, 8.18, 8.21, 8.38, 8.98, 9.46, $9.54,9.59,9.77,9.94,11.18,11.52,11.59,11.65,11.88$, $11.94,11.99,12.07,12.53,12.66,12.66,13.07,14.55$, $16.00,16.95,17.56,17.7,18.17,19.17,19.21,19.66$, $21.13,21.66,22.41,23.43,26.21$, and 32.76 .

(ii) The Second Progressively Type II Censored Sample Suppose $m=43$ and then apply the following to the second dataset,

$$
\begin{aligned}
& R_{i}=1, \quad i=1, \ldots, 20, \\
& R_{i}=0, \quad i=21, \ldots, 43 .
\end{aligned}
$$

Then the second progressively type II censored sample is $0.55,0.77,0.84,0.93,1.11,1.25,1.27,1.29$, $1.36,1.42,1.49,1.49,1.50,1.52,1.54,1.58,1.59,1.61$,
$1.61,1.62,1.64,1.66,1.66,1.66,1.67,1.68,1.68,1.69$, $1.70,1.70,1.73,1.76,1.76,1.77,1.78,1.81,1.82,1.84$, $1.84,1.89,2.00,2.01$, and 2.24 . For the two progressively type II censored samples mentioned above, the ML, M, LS, and WLS estimates of the parameters $\eta, \sigma$, and $\theta$ are computed and presented in Table 3.

\section{Simulation Procedure}

In what follows, estimations using the ML, M, LS, and WLS methods for the parameters $\eta, \sigma$, and $\theta$ are computed and compared using a Monte Carlo simulation according to the following steps:

(1) According to the algorithm given in Balakrishnan and Sandhu [26], generate a progressively type II censored sample of size $m$ from the EDPD with CDF (4).

(2) The ML, M, LS, and WLS estimates of the parameters $\eta, \sigma$, and $\theta$ are computed as shown in Section 3.

(3) The above steps are repeated 5,000 times.

(4) Let $\widehat{\Lambda}$ be an estimate of $\Lambda$. Then the estimates average, mean squared error (MSE), and relative absolute bias (RAB) of $\widehat{\Lambda}$ over the 5,000 samples are given, respectively, by

$$
\begin{aligned}
\bar{\Lambda} & =\frac{1}{5,000} \sum_{i=1}^{5,000} \widehat{\Lambda}_{i}, \\
\operatorname{MSE}(\widehat{\Lambda}) & =\frac{1}{5,000} \sum_{i=1}^{5,000}\left(\widehat{\Lambda}_{i}-\Lambda\right)^{2}, \\
\operatorname{RAB}(\widehat{\Lambda}) & =\frac{1}{5,000} \sum_{i=1}^{5,000} \frac{\left|\widehat{\Lambda}_{i}-\Lambda\right|}{\Lambda} .
\end{aligned}
$$

(5) As shown in Step 4, calculate the estimates average of the parameters $\eta, \sigma$, and $\theta$ and their MSEs and RABs. Calculate also the mean of the MSEs (MMSE) and the mean of the RABs (MRAB) using the next two relations:

$$
\begin{aligned}
\operatorname{MMSE} & =\frac{\operatorname{MSE}(\widehat{\eta})+\operatorname{MSE}(\widehat{\sigma})+\operatorname{MSE}(\widehat{\theta})}{3}, \\
\operatorname{MRAB} & =\frac{\operatorname{RAB}(\widehat{\eta})+\operatorname{RAB}(\widehat{\sigma})+\operatorname{RAB}(\widehat{\theta})}{3} .
\end{aligned}
$$

The computational results are presented in Tables 4 and 5. The following four CSs are used through generating the samples:

(i) CS1:

$$
\begin{array}{ll}
R_{i}=1, & i=1, \ldots, n-m, \\
R_{i}=0, & \text { otherwise, }
\end{array}
$$


TABle 1: Some descriptive statistics for the two datasets.

\begin{tabular}{|c|c|c|c|c|c|c|c|c|c|c|}
\hline Data & Mode (s) & Median & Mean & Variance & $\mathrm{CV}$ & Skewness & Kurtosis & Range & Min & Max \\
\hline 1 & $\begin{array}{cc}7.22 & 11.99 \\
12.66 & 22.41 \\
\end{array}$ & 12.48 & 14.888 & 52.838 & 0.488 & 1.052 & 3.623 & 33.61 & 4.35 & 37.96 \\
\hline 2 & 1.61 & 1.59 & 1.507 & 0.105 & 0.215 & -0.9 & 3.924 & 1.69 & 0.55 & 2.24 \\
\hline
\end{tabular}

$\mathrm{CV}$ : coefficient of variation.

TABle 2: The MLEs, K-S statistic, $p$ value, AIC, CAIC, and BIC for the two datasets.

\begin{tabular}{|c|c|c|c|c|c|c|c|c|c|}
\hline \multicolumn{10}{|c|}{ The first dataset } \\
\hline Model & $\eta$ & $\sigma$ & $\theta$ & $\hat{\gamma}$ & $\mathrm{K}-\mathrm{S}$ & $p$ value & AIC & CAIC & $\mathrm{BIC}$ \\
\hline EDPD & 13.6983 & 11.8965 & 0.0219 & - & 0.0728 & 0.75786 & 559.9 & 560.242 & 567.3 \\
\hline EEPD & 1.6066 & 3.1671 & 0.0905 & - & 0.1208 & 0.16727 & 573.6 & 573.899 & 580.9 \\
\hline EWPD & 3.3840 & 1.4609 & 1.0781 & 0.3567 & 0.4733 & $2.20 \times 10^{-13}$ & 700.4 & 700.7 & 707.7 \\
\hline PED & 0.5305 & - & 0.0741 & - & 0.2908 & $1.10 \times 10^{-6}$ & 624.6 & 624.7 & 629.4 \\
\hline EPD & - & 0.0038 & 0.0677 & - & 0.3267 & $2.60 \times 10^{-8}$ & 633.2 & 633.3 & 638.0 \\
\hline ED & - & - & 0.0672 & - & 0.3241 & $3.50 \times 10^{-8}$ & 631.1 & 631.1 & 633.5 \\
\hline \multicolumn{10}{|c|}{ The second dataset } \\
\hline EDPD & 34.838 & 0.4619 & 2.4403 & - & 0.1872 & 0.0242 & 68.56 & 68.97 & 74.99 \\
\hline EEPD & 6.6783 & 4.9030 & 0.6944 & - & 0.2368 & 0.0017 & 78.81 & 79.22 & 85.24 \\
\hline EWPD & 0.7592 & 2.6799 & 0.9182 & 1.5689 & 0.2491 & 0.0008 & 80.73 & 81.13 & 87.16 \\
\hline PED & 9.8176 & - & 1.9320 & - & 0.2728 & 0.0002 & 84.86 & 85.06 & 89.14 \\
\hline EPD & - & 0.0181 & 0.7267 & - & 0.4398 & $5.20 \times 10^{-11}$ & 182.6 & 182.8 & 186.8 \\
\hline ED & - & - & 0.6636 & - & 0.4046 & $2.20 \times 10^{-9}$ & 179.7 & 179.7 & 181.8 \\
\hline
\end{tabular}

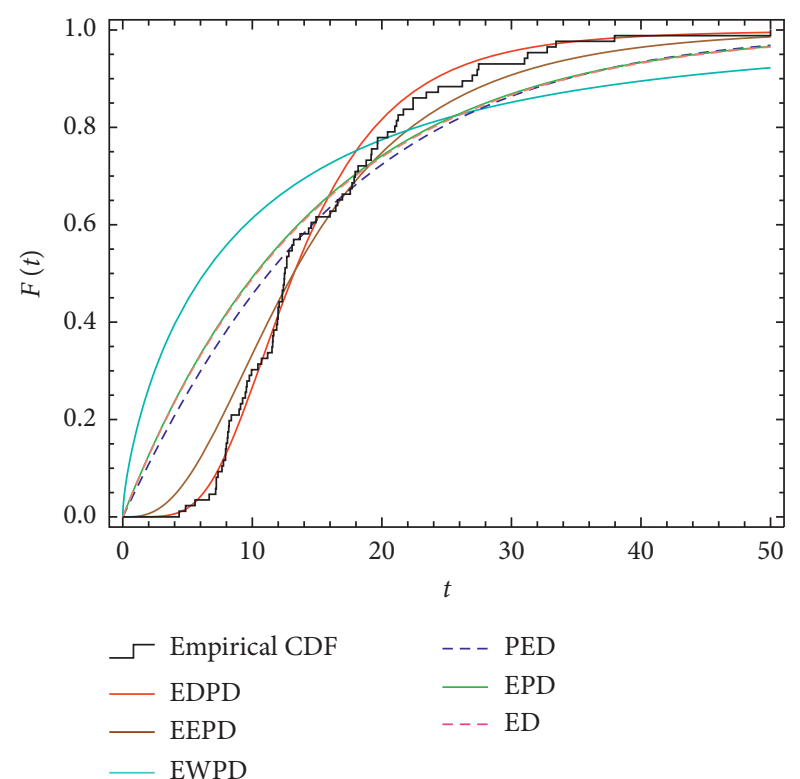

(a)

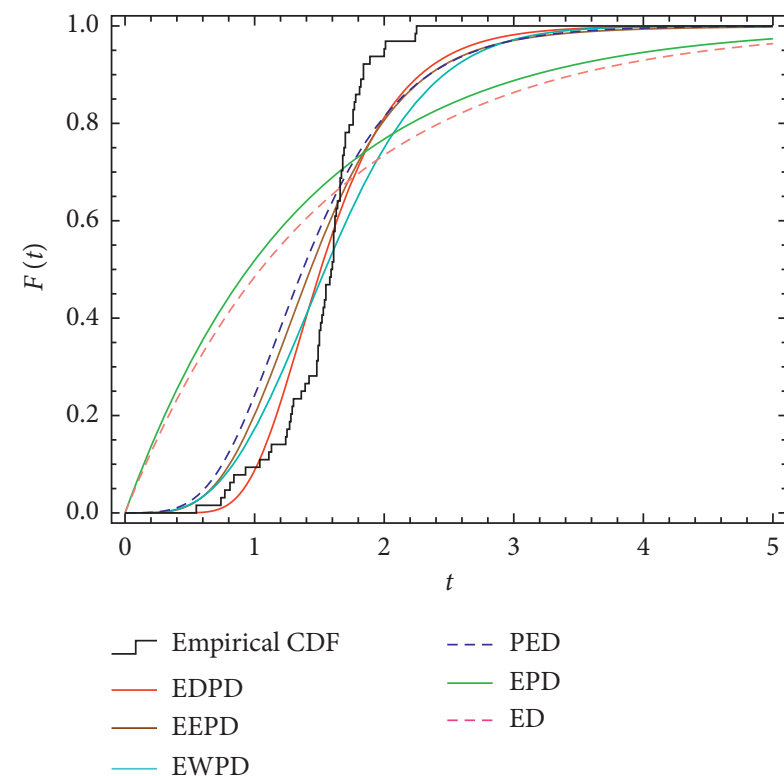

(b)

Figure 6: Empirical CDF versus CDF of EDPD, EWPD, EEPD, EPD, PED, and ED for the (a) first dataset and (b) second dataset.

TABLE 3: Estimations using the ML, M, LS, and WLS methods for the parameters $\eta, \sigma$, and $\theta$.

\begin{tabular}{lccccr}
\hline & \multicolumn{3}{c}{ The first censored sample } & \multicolumn{3}{c}{ The second censored sample } \\
Methods & $\hat{\eta}$ & $\hat{\sigma}$ & $\hat{\theta}$ & $\hat{\eta}$ & $\hat{\sigma}$ \\
\hline ML & 14.2867 & 17.3397 & 0.014948 & 36.1439 & 0.496282 \\
M & 21.1755 & 13.2365 & 0.027392 & 45.5460 & 1.054180 \\
LS & 13.1991 & 8.71130 & 0.031710 & 57.8003 & 0.234002 \\
WLS & 15.0092 & 9.28229 & 0.031072 & 36.5016 & 0.278964 \\
\hline
\end{tabular}


TABLE 4: ML and M estimates of $\eta, \sigma$, and $\theta$ with their MSEs, RABs, MMSE, and MRAB based on 5000 simulations. Population parameter values are $\eta=4.0, \sigma=4.0$, and $\theta=1.5$.

\begin{tabular}{|c|c|c|c|c|c|c|c|c|c|c|}
\hline \multirow[b]{2}{*}{$n$} & \multirow[b]{2}{*}{$m$} & \multirow[b]{2}{*}{ CS } & \multicolumn{4}{|c|}{ MLE } & \multicolumn{4}{|c|}{$\mathrm{ME}$} \\
\hline & & & $\begin{array}{l}\overline{\widehat{\eta}}_{M L} \\
\overline{\widehat{\sigma}}_{M L} \\
\widehat{\hat{\theta}}_{M L} \\
\end{array}$ & $\begin{array}{l}\operatorname{MSE}\left(\widehat{\eta}_{M L}\right) \\
\operatorname{MSE}\left(\widehat{\sigma}_{M L}\right) \\
\operatorname{MSE}\left(\widehat{\theta}_{M L}\right)\end{array}$ & $\begin{array}{l}\operatorname{RAB}\left(\widehat{\eta}_{M L}\right) \\
\operatorname{RAB}\left(\widehat{\sigma}_{M L}\right) \\
\operatorname{RAB}\left(\widehat{\theta}_{M L}\right)\end{array}$ & $\begin{array}{l}\text { MMSE } \\
\text { MRAB }\end{array}$ & $\begin{array}{l}\overline{\widehat{\eta}}_{M} \\
\overline{\widehat{\sigma}}_{M} \\
\widehat{\hat{\theta}}_{M} \\
\end{array}$ & $\begin{array}{l}\operatorname{MSE}\left(\widehat{\eta}_{M}\right) \\
\operatorname{MSE}\left(\widehat{\sigma}_{M}\right) \\
\operatorname{MSE}\left(\widehat{\theta}_{M}\right)\end{array}$ & $\begin{array}{l}\operatorname{RAB}\left(\widehat{\eta}_{M}\right) \\
\operatorname{RAB}\left(\widehat{\sigma}_{M}\right) \\
\operatorname{RAB}\left(\widehat{\theta}_{M}\right)\end{array}$ & $\begin{array}{l}\text { MMSE } \\
\text { MRAB }\end{array}$ \\
\hline \multirow{27}{*}{25} & \multirow{13}{*}{15} & \multirow{3}{*}{1} & 4.4880 & 4.0099 & 0.3820 & 2.0052 & 3.7856 & 5.5707 & 0.4311 & 5.4257 \\
\hline & & & 4.2135 & 1.7027 & 0.2206 & 0.2950 & 1.7172 & 7.0330 & 0.6139 & 0.6563 \\
\hline & & & 1.5684 & 0.3031 & 0.2825 & & 2.8680 & 3.6736 & 0.9240 & \\
\hline & & \multirow{4}{*}{2} & 4.4683 & 4.2749 & 0.3922 & 2.0734 & 4.0441 & 4.9030 & 0.4390 & 4.5112 \\
\hline & & & 4.2713 & 1.6678 & 0.2252 & 0.2953 & 2.2982 & 5.6220 & 0.5256 & 0.5902 \\
\hline & & & 1.5267 & 0.2776 & 0.2685 & & 2.6678 & 3.0086 & 0.8061 & \\
\hline & & & 4.5508 & 4.1540 & 0.3845 & 2.0262 & 3.8039 & 5.9571 & 0.4854 & 5.2455 \\
\hline & & \multirow[t]{3}{*}{3} & 4.1972 & 1.5927 & 0.2192 & 0.2982 & 1.9483 & 6.8558 & 0.6093 & 0.6368 \\
\hline & & & 1.5934 & 0.3318 & 0.2908 & & 2.6865 & 2.9236 & 0.8157 & \\
\hline & & & 4.5078 & 4.1658 & 0.3868 & 2.0032 & 4.4974 & 9.1003 & 0.5329 & 6.4233 \\
\hline & & \multirow[t]{3}{*}{4} & 4.1923 & 1.5320 & 0.2171 & 0.2960 & 1.9950 & 6.3103 & 0.5592 & 0.6807 \\
\hline & & & 1.5723 & 0.3117 & 0.2840 & & 2.9084 & 3.8593 & 0.9500 & \\
\hline & & & 4.4747 & 3.9587 & 0.3763 & 2.0006 & 3.7620 & 4.5589 & 0.4111 & 4.0868 \\
\hline & \multirow{12}{*}{20} & \multirow[t]{3}{*}{1} & 4.2838 & 1.7601 & 0.2350 & 0.2946 & 2.1579 & 4.9121 & 0.4830 & 0.5424 \\
\hline & & & 1.5406 & 0.2831 & 0.2724 & & 2.5663 & 2.7893 & 0.7332 & \\
\hline & & & 4.4206 & 3.7027 & 0.3644 & 1.9083 & 3.5770 & 4.0648 & 0.4008 & 3.6891 \\
\hline & & \multirow[t]{3}{*}{2} & 4.2969 & 1.7528 & 0.2340 & 0.2877 & 2.1758 & 4.8170 & 0.4819 & 0.5192 \\
\hline & & & 1.5139 & 0.2694 & 0.2648 & & 2.4660 & 2.1854 & 0.6751 & \\
\hline & & & 4.5069 & 3.9603 & 0.3743 & 1.9984 & 3.9556 & 6.9133 & 0.4834 & 5.0598 \\
\hline & & \multirow[t]{3}{*}{3} & 4.3009 & 1.7607 & 0.2324 & 0.2915 & 2.0338 & 5.5173 & 0.5235 & 0.5833 \\
\hline & & & 1.5296 & 0.2741 & 0.2679 & & 2.5805 & 2.7488 & 0.7428 & \\
\hline & & & 4.5106 & 3.9751 & 0.3756 & 1.9693 & 3.8776 & 6.2540 & 0.4743 & 4.9159 \\
\hline & & 4 & 4.2642 & 1.6679 & 0.2303 & 0.2901 & 2.0042 & 5.5938 & 0.5255 & 0.5883 \\
\hline & & & 1.5435 & 0.2650 & 0.2644 & & 2.6154 & 2.8999 & 0.7649 & \\
\hline & & & 4.4320 & 3.6128 & 0.3584 & 1.9000 & 3.7933 & 5.2457 & 0.442 & 3.6996 \\
\hline & 25 & - & 4.3522 & 1.8298 & 0.2429 & 0.2878 & 2.4837 & 3.9823 & 0.4186 & 0.4827 \\
\hline & & & 1.4997 & 0.2574 & 0.2621 & & 2.3169 & 1.8708 & 0.5874 & \\
\hline & & & 4.2797 & 2.3832 & 0.2897 & 1.4005 & 3.2276 & 2.4115 & 0.3191 & 2.8905 \\
\hline & & 1 & 4.2911 & 1.5536 & 0.2228 & 0.2585 & 2.1191 & 4.7406 & 0.4803 & 0.4592 \\
\hline & & & 1.5210 & 0.2646 & 0.2629 & & 2.3080 & 1.5195 & 0.5783 & \\
\hline & & & 4.2262 & 2.3459 & 0.2909 & 1.4124 & 3.5641 & 4.2177 & 0.4038 & 3.3436 \\
\hline & & 2 & 4.3059 & 1.6422 & 0.2301 & 0.2595 & 2.4131 & 4.0116 & 0.4301 & 0.4677 \\
\hline & & & 1.5004 & 0.2490 & 0.2576 & & 2.2752 & 1.8015 & 0.5692 & \\
\hline & 30 & & 4.2754 & 2.3806 & 0.2858 & 1.4292 & 2.8691 & 2.9306 & 0.3594 & 3.1818 \\
\hline & & 3 & 4.3016 & 1.6340 & 0.2290 & 0.2601 & 2.1157 & 5.0001 & 0.4925 & 0.4724 \\
\hline & & & 1.5232 & 0.2731 & 0.2655 & & 2.2889 & 1.6147 & 0.5654 & \\
\hline & & & 4.2922 & 2.5213 & 0.2959 & 1.4527 & 3.2683 & 4.5953 & 0.4184 & 3.5313 \\
\hline & & 4 & 4.2638 & 1.5854 & 0.2264 & 0.2599 & 2.1790 & 4.5737 & 0.4676 & 0.4736 \\
\hline & & & 1.5247 & 0.2515 & 0.2573 & & 2.2463 & 1.4249 & 0.5348 & \\
\hline & & & 4.2097 & 2.0664 & 0.2715 & 1.3102 & 3.7420 & 4.0931 & 0.3829 & 3.1515 \\
\hline 50 & & 1 & 4.3314 & 1.6268 & 0.2345 & 0.2531 & 2.4818 & 3.9559 & 0.4231 & 0.4502 \\
\hline & & & 1.4866 & 0.2374 & 0.2532 & & 2.2267 & 1.4056 & 0.5447 & \\
\hline & & & 4.2036 & 2.2132 & 0.2794 & 1.3507 & 4.8363 & 7.7696 & 0.5400 & 3.9701 \\
\hline & & 2 & 4.3227 & 1.5991 & 0.2338 & 0.2555 & 2.9342 & 3.0713 & 0.3647 & 0.4682 \\
\hline & & & 1.4841 & 0.2397 & 0.2534 & & 2.1884 & 1.0693 & 0.4997 & \\
\hline & 40 & & 4.2456 & 2.1781 & 0.2767 & 1.3533 & 3.9856 & 4.5764 & 0.4292 & 3.6425 \\
\hline & & 3 & 4.3459 & 1.6397 & 0.2350 & 0.2547 & 2.2268 & 4.5080 & 0.4695 & 0.5280 \\
\hline & & & 1.4944 & 0.2420 & 0.2525 & & 2.4627 & 1.8430 & 0.6853 & \\
\hline & & & 4.1935 & 2.1201 & 0.2747 & 1.3267 & 4.4829 & 6.1933 & 0.4772 & 3.8678 \\
\hline & & 4 & 4.3382 & 1.6128 & 0.2339 & 0.2557 & 2.4408 & 3.8008 & 0.4204 & 0.5052 \\
\hline & & & 1.4867 & 0.2472 & 0.2585 & & 2.3681 & 1.6091 & 0.6179 & \\
\hline & & & 4.1714 & 1.8511 & 0.2543 & 1.2189 & 2.3396 & 3.5492 & 0.4256 & 1.7983 \\
\hline & 50 & - & 4.3256 & 1.5803 & 0.2343 & 0.2451 & 3.2250 & 1.6318 & 0.2321 & 0.2965 \\
\hline & & & 1.4731 & 0.2254 & 0.2468 & & 1.8108 & 0.2139 & 0.2318 & \\
\hline
\end{tabular}


TABle 4: Continued.

\begin{tabular}{|c|c|c|c|c|c|c|c|c|c|c|}
\hline \multirow[b]{2}{*}{$n$} & \multirow[b]{2}{*}{$m$} & \multirow[b]{2}{*}{ CS } & \multicolumn{4}{|c|}{ MLE } & \multicolumn{4}{|c|}{ ME } \\
\hline & & & $\begin{array}{l}\overline{\widehat{\eta}}_{M L} \\
\widehat{\widehat{\sigma}}_{M L} \\
\widehat{\theta}_{M L}\end{array}$ & $\begin{array}{l}\operatorname{MSE}\left(\widehat{\eta}_{M L}\right) \\
\operatorname{MSE}\left(\widehat{\sigma}_{M L}\right) \\
\operatorname{MSE}\left(\widehat{\theta}_{M L}\right)\end{array}$ & $\begin{array}{l}\operatorname{RAB}\left(\widehat{\eta}_{M L}\right) \\
\operatorname{RAB}\left(\widehat{\sigma}_{M L}\right) \\
\operatorname{RAB}\left(\widehat{\theta}_{M L}\right)\end{array}$ & $\begin{array}{l}\text { MMSE } \\
\text { MRAB }\end{array}$ & $\begin{array}{l}\overline{\widehat{\eta}}_{M} \\
\widehat{\widehat{\sigma}}_{M} \\
\widehat{\theta}_{M}\end{array}$ & $\begin{array}{l}\operatorname{MSE}\left(\widehat{\eta}_{M}\right) \\
\operatorname{MSE}\left(\widehat{\sigma}_{M}\right) \\
\operatorname{MSE}\left(\widehat{\theta}_{M}\right)\end{array}$ & $\begin{array}{l}\operatorname{RAB}\left(\widehat{\eta}_{M}\right) \\
\operatorname{RAB}\left(\widehat{\sigma}_{M}\right) \\
\operatorname{RAB}\left(\widehat{\theta}_{M}\right)\end{array}$ & $\begin{array}{l}\text { MMSE } \\
\text { MRAB }\end{array}$ \\
\hline \multirow{27}{*}{100} & \multirow{13}{*}{60} & \multirow{4}{*}{1} & 4.0679 & 1.0237 & 0.1935 & 0.8853 & 4.2069 & 1.8942 & 0.2429 & 1.7737 \\
\hline & & & 4.3006 & 1.4184 & 0.2190 & 0.2177 & 2.9985 & 2.3610 & 0.2956 & 0.3455 \\
\hline & & & 1.4846 & 0.2139 & 0.2408 & & 2.1522 & 1.0658 & 0.4980 & \\
\hline & & & 4.0723 & 1.2257 & 0.2144 & 0.9897 & 3.6262 & 1.5438 & 0.1878 & 0.9533 \\
\hline & & \multirow[t]{3}{*}{2} & 4.3519 & 1.5280 & 0.2330 & 0.2305 & 4.0883 & 0.9290 & 0.1534 & 0.1950 \\
\hline & & & 1.4621 & 0.2155 & 0.2442 & & 1.8265 & 0.3872 & 0.2438 & \\
\hline & & & 4.0846 & 1.0849 & 0.2024 & 0.9121 & 4.1135 & 0.0657 & 0.0306 & 0.3085 \\
\hline & & \multirow[t]{3}{*}{3} & 4.2877 & 1.4374 & 0.2204 & 0.2207 & 4.1716 & 0.4981 & 0.0835 & 0.1068 \\
\hline & & & 1.4875 & 0.2139 & 0.2392 & & 1.8015 & 0.3616 & 0.2062 & \\
\hline & & & 4.0722 & 1.1576 & 0.2074 & 0.9439 & 3.9421 & 0.2701 & 0.0439 & 0.5029 \\
\hline & & \multirow[t]{3}{*}{4} & 4.2924 & 1.4558 & 0.2233 & 0.2237 & 4.3331 & 0.8608 & 0.1266 & 0.1121 \\
\hline & & & 1.4872 & 0.2182 & 0.2405 & & 1.7023 & 0.3779 & 0.1657 & \\
\hline & & & 4.0469 & 0.9291 & 0.1868 & 0.8844 & 3.4705 & 1.7272 & 0.2219 & 1.2650 \\
\hline & \multirow{11}{*}{80} & \multirow[t]{3}{*}{1} & 4.3393 & 1.5213 & 0.2301 & 0.2184 & 4.3732 & 1.5375 & 0.1667 & 0.2234 \\
\hline & & & 1.4645 & 0.2027 & 0.2382 & & 1.8741 & 0.5303 & 0.2817 & \\
\hline & & & 4.0082 & 0.9902 & 0.1924 & 0.8988 & 4.1911 & 1.6957 & 0.2111 & 0.9800 \\
\hline & & \multirow[t]{3}{*}{2} & 4.3153 & 1.4993 & 0.2310 & 0.2206 & 4.6798 & 1.0861 & 0.1984 & 0.1935 \\
\hline & & & 1.4609 & 0.2070 & 0.2384 & & 1.7341 & 0.1582 & 0.1709 & \\
\hline & & & 4.0811 & 0.9167 & 0.1850 & 0.8528 & 4.0304 & 0.3209 & 0.0476 & 0.5693 \\
\hline & & \multirow[t]{3}{*}{3} & 4.3001 & 1.4320 & 0.2240 & 0.2152 & 4.7319 & 1.1710 & 0.1984 & 0.1256 \\
\hline & & & 1.4836 & 0.2097 & 0.2365 & & 1.6055 & 0.2160 & 0.1308 & \\
\hline & & & 4.0452 & 0.9568 & 0.1886 & 0.8791 & 3.7794 & 1.0120 & 0.1352 & 0.7997 \\
\hline & & \multirow[t]{3}{*}{4} & 4.3120 & 1.4746 & 0.2262 & 0.2175 & 4.5011 & 1.0398 & 0.1450 & 0.1532 \\
\hline & & & 1.4680 & 0.2059 & 0.2375 & & 1.7367 & 0.3474 & 0.1793 & \\
\hline & \multirow{3}{*}{100} & & 4.0063 & 0.8184 & 0.1752 & 0.8269 & 3.2165 & 0.8785 & 0.2044 & 0.4700 \\
\hline & & \multirow[t]{2}{*}{-} & 4.3327 & 1.4665 & 0.2270 & 0.2115 & 4.5539 & 0.4004 & 0.1394 & 0.1611 \\
\hline & & & 1.4543 & 0.1957 & 0.2323 & & 1.7082 & 0.1312 & 0.1395 & \\
\hline
\end{tabular}

which means we eliminate one unit after every failure observed in the first $n-m$ failures in the sample.

(ii) $\mathrm{CS} 2$ :

$$
\begin{aligned}
& R_{i}=n-m, \quad i=1, \\
& R_{i}=0, \quad \text { otherwise, }
\end{aligned}
$$

which means we eliminate $n-m$ units after the first failure observed in the sample.

(iii) CS3:

$$
\begin{aligned}
& R_{i}=n-m, \quad i=\frac{m+1}{2}(m \text { odd }) \text { or } i=\frac{m}{2}(m \text { even }), \\
& R_{i}=0, \quad \text { otherwise, }
\end{aligned}
$$

which means we eliminate $n-m$ units after the middle failure observed in the sample. (iv) CS4:

$$
\begin{aligned}
& R_{i}=\left[\frac{3}{5}(n-m)\right], \quad i=1, \\
& R_{i}=n-m-\left[\frac{3}{5}(n-m)\right] \quad i=\frac{m+1}{2} \text { (modd) or } i=\frac{m}{2}(\text { meven }), \\
& R_{i}=0, \quad \text { otherwise, }
\end{aligned}
$$

which means we eliminate $[(3 / 5)(n-m)]$ units after the first observed failure and eliminate $n-m-$ $[(3 / 5)(n-m)]$ units after the middle failure observed in the sample. The $[z]$ indicates the greatest integer less than or equal to $z$.

Different values of $m(=60 \%, 80 \%$, and $100 \%$ of the sample size) have been considered through the simulation procedure. The values of the population parameters are $\eta=4.0, \sigma=4.0$, and $\theta=1.5$.

It can be noticed that the WLS estimates are better than the LS, ML, and M estimates via the MMSEs and MRABs, 
TABLE 5: LS and WLS estimates of $\eta, \sigma$, and $\theta$ with their MSEs, RABs, MMSE, and MRAB based on 5, 000 simulations. Population parameter values are $\eta=4.0, \sigma=4.0$, and $\theta=1.5$.

\begin{tabular}{|c|c|c|c|c|c|c|c|c|c|c|}
\hline \multirow[b]{2}{*}{$n$} & \multirow[b]{2}{*}{$m$} & \multirow[b]{2}{*}{ CS } & \multicolumn{4}{|c|}{ LSE } & \multicolumn{4}{|c|}{ WLSE } \\
\hline & & & $\begin{array}{l}\overline{\hat{\eta}}_{L} \\
\overline{\widehat{\sigma}}_{L} \\
\widehat{\widehat{\theta}}_{L} \\
\end{array}$ & $\begin{array}{l}\operatorname{MSE}\left(\widehat{\eta}_{L}\right) \\
\operatorname{MSE}\left(\widehat{\sigma}_{L}\right) \\
\operatorname{MSE}\left(\widehat{\theta}_{L}\right)\end{array}$ & $\begin{array}{l}\operatorname{RAB}\left(\widehat{\eta}_{L}\right) \\
\operatorname{RAB}\left(\widehat{\sigma}_{L}\right) \\
\operatorname{RAB}\left(\widehat{\theta}_{L}\right)\end{array}$ & $\begin{array}{l}\text { MMSE } \\
\text { MRAB }\end{array}$ & $\begin{array}{l}\overline{\hat{\eta}}_{W} \\
\overline{\widehat{\sigma}}_{W} \\
\widehat{\hat{\theta}}_{W} \\
\end{array}$ & $\begin{array}{l}\operatorname{MSE}\left(\hat{\eta}_{W}\right) \\
\operatorname{MSE}\left(\widehat{\sigma}_{W}\right) \\
\operatorname{MSE}\left(\widehat{\theta}_{W}\right)\end{array}$ & $\begin{array}{l}\operatorname{RAB}\left(\widehat{\eta}_{W}\right) \\
\operatorname{RAB}\left(\widehat{\sigma}_{W}\right) \\
\operatorname{RAB}\left(\widehat{\theta}_{W}\right)\end{array}$ & $\begin{array}{l}\text { MMSE } \\
\text { MRAB }\end{array}$ \\
\hline \multirow{27}{*}{25} & \multirow{13}{*}{15} & \multirow{3}{*}{1} & 4.2018 & 3.8547 & 0.3684 & 2.1198 & 4.2775 & 3.2795 & 0.3373 & 1.8863 \\
\hline & & & 4.1913 & 2.1909 & 0.2254 & 0.2934 & 4.1459 & 2.0644 & 0.2153 & 0.2790 \\
\hline & & & 1.5048 & 0.3138 & 0.2864 & & 1.5483 & 0.3149 & 0.2843 & \\
\hline & & \multirow{4}{*}{2} & 4.2342 & 4.3133 & 0.3810 & 2.3266 & 4.3473 & 3.6601 & 0.3512 & 2.0175 \\
\hline & & & 4.2218 & 2.3387 & 0.2282 & 0.2956 & 4.2696 & 2.1016 & 0.2138 & 0.2793 \\
\hline & & & 1.4854 & 0.3277 & 0.2775 & & 1.4957 & 0.2910 & 0.2728 & \\
\hline & & & 4.2297 & 3.8954 & 0.3672 & 2.1470 & 4.2814 & 3.3869 & 0.3391 & 1.8355 \\
\hline & & \multirow[t]{3}{*}{3} & 4.1701 & 2.1944 & 0.2239 & 0.2955 & 4.0525 & 1.8140 & 0.2084 & 0.2760 \\
\hline & & & 1.5232 & 0.3511 & 0.2955 & & 1.5678 & 0.3057 & 0.2804 & \\
\hline & & & 4.2309 & 4.1101 & 0.3788 & 2.2403 & 4.3207 & 3.5594 & 0.3468 & 2.0196 \\
\hline & & \multirow[t]{3}{*}{4} & 4.1892 & 2.2634 & 0.2261 & 0.3004 & 4.2193 & 2.1714 & 0.2175 & 0.2850 \\
\hline & & & 1.5150 & 0.3473 & 0.2965 & & 1.5332 & 0.3279 & 0.2909 & \\
\hline & & & 4.2268 & 3.5707 & 0.3461 & 2.0226 & 4.3054 & 3.0713 & 0.3216 & 1.7824 \\
\hline & \multirow{12}{*}{20} & \multirow[t]{3}{*}{1} & 4.2142 & 2.2062 & 0.2190 & 0.2762 & 4.2661 & 2.0199 & 0.2065 & 0.2610 \\
\hline & & & 1.4944 & 0.2908 & 0.2635 & & 1.4944 & 0.2559 & 0.2548 & \\
\hline & & & 4.1998 & 3.5906 & 0.3535 & 1.9432 & 4.2734 & 3.0718 & 0.3275 & 1.7414 \\
\hline & & \multirow[t]{3}{*}{2} & 4.1557 & 1.9528 & 0.2072 & 0.2734 & 4.2575 & 1.8905 & 0.2015 & 0.2618 \\
\hline & & & 1.4975 & 0.2864 & 0.2594 & & 1.4821 & 0.2619 & 0.2564 & \\
\hline & & & 4.1763 & 3.5149 & 0.3488 & 1.9955 & 4.2382 & 2.9586 & 0.3211 & 1.6911 \\
\hline & & \multirow[t]{3}{*}{3} & 4.1589 & 2.1746 & 0.2205 & 0.2785 & 4.1836 & 1.8553 & 0.2036 & 0.2606 \\
\hline & & & 1.503 & 0.2970 & 0.2661 & & 1.5058 & 0.2595 & 0.2573 & \\
\hline & & & 4.2168 & 3.6427 & 0.3497 & 2.0747 & 4.2818 & 3.0952 & 0.3228 & 1.7780 \\
\hline & & 4 & 4.2207 & 2.3000 & 0.2227 & 0.2780 & 4.2651 & 1.9773 & 0.2046 & 0.2619 \\
\hline & & & 1.4914 & 0.2814 & 0.2617 & & 1.4896 & 0.2617 & 0.2584 & \\
\hline & & & 4.1975 & 3.2876 & 0.3299 & 1.8774 & 4.2474 & 2.8569 & 0.3113 & 1.6443 \\
\hline & 25 & - & 4.1717 & 2.0857 & 0.2076 & 0.2609 & 4.2778 & 1.8419 & 0.2000 & 0.2517 \\
\hline & & & 1.4959 & 0.2589 & 0.2453 & & 1.4671 & 0.2340 & 0.2439 & \\
\hline & & & 4.1671 & 2.6825 & 0.3021 & 1.5410 & 4.2294 & 2.1876 & 0.2712 & 1.2432 \\
\hline & & 1 & 4.1100 & 1.6989 & 0.1938 & 0.2473 & 4.0173 & 1.3254 & 0.1771 & 0.2282 \\
\hline & & & 1.5239 & 0.2416 & 0.2460 & & 1.5694 & 0.2165 & 0.2364 & \\
\hline & & & 4.1593 & 2.8978 & 0.3113 & 1.6367 & 4.2344 & 2.3133 & 0.2796 & 1.3704 \\
\hline & & 2 & 4.1470 & 1.7885 & 0.1963 & 0.2460 & 4.2121 & 1.5797 & 0.1868 & 0.2327 \\
\hline & & & 1.4955 & 0.2239 & 0.2304 & & 1.4941 & 0.2181 & 0.2318 & \\
\hline & 30 & & 4.1583 & 2.8172 & 0.3105 & 1.5909 & 4.1947 & 2.0972 & 0.2699 & 1.2279 \\
\hline & & 3 & 4.0803 & 1.7078 & 0.1909 & 0.2501 & 4.0203 & 1.3665 & 0.1819 & 0.2304 \\
\hline & & & 1.5267 & 0.2477 & 0.2490 & & 1.5576 & 0.2199 & 0.2394 & \\
\hline & & & 4.1557 & 2.8545 & 0.3093 & 1.6398 & 4.2266 & 2.2891 & 0.2791 & 1.3457 \\
\hline & & 4 & 4.0962 & 1.8139 & 0.1979 & 0.2501 & 4.1001 & 1.5258 & 0.1832 & 0.2340 \\
\hline & & & 1.5214 & 0.2511 & 0.2430 & & 1.5369 & 0.2222 & 0.2397 & \\
\hline & & & 4.1554 & 2.2927 & 0.2774 & 1.4242 & 4.2138 & 1.8709 & 0.2555 & 1.1424 \\
\hline 50 & & 1 & 4.1277 & 1.7714 & 0.1888 & 0.2281 & 4.1709 & 1.3666 & 0.1750 & 0.2160 \\
\hline & & & 1.5167 & 0.2086 & 0.2179 & & 1.5102 & 0.1897 & 0.2176 & \\
\hline & & & 4.1382 & 2.3300 & 0.2819 & 1.4075 & 4.2014 & 1.9425 & 0.2584 & 1.1516 \\
\hline & & 2 & 4.1258 & 1.6930 & 0.1900 & 0.2302 & 4.1836 & 1.3258 & 0.1755 & 0.2170 \\
\hline & & & 1.5049 & 0.1995 & 0.2187 & & 1.4946 & 0.1866 & 0.2170 & \\
\hline & 40 & & 4.1329 & 2.3980 & 0.2853 & 1.4030 & 4.1742 & 1.9651 & 0.2573 & 1.1994 \\
\hline & & 3 & 4.1038 & 1.6115 & 0.1876 & 0.2320 & 4.1378 & 1.4478 & 0.1790 & 0.2182 \\
\hline & & & 1.5131 & 0.1996 & 0.2231 & & 1.5123 & 0.1854 & 0.2183 & \\
\hline & & & 4.1497 & 2.4247 & 0.2868 & 1.4009 & 4.2040 & 2.0314 & 0.2632 & 1.1723 \\
\hline & & 4 & 4.1246 & 1.5883 & 0.1843 & 0.2293 & 4.1606 & 1.2879 & 0.1724 & 0.2192 \\
\hline & & & 1.5057 & 0.1898 & 0.2168 & & 1.5079 & 0.1977 & 0.2221 & \\
\hline & & & 4.1168 & 2.0442 & 0.2584 & 1.2361 & 4.1589 & 1.7465 & 0.2426 & 1.0439 \\
\hline & 50 & - & 4.1196 & 1.4875 & 0.1748 & 0.2120 & 4.1824 & 1.2104 & 0.1684 & 0.2058 \\
\hline & & & 1.5028 & 0.1766 & 0.2028 & & 1.4867 & 0.1749 & 0.2065 & \\
\hline
\end{tabular}


TABLE 5: Continued.

\begin{tabular}{|c|c|c|c|c|c|c|c|c|c|c|}
\hline \multirow[b]{2}{*}{$n$} & \multirow[b]{2}{*}{$m$} & \multirow[b]{2}{*}{ CS } & \multicolumn{4}{|c|}{ LSE } & \multicolumn{4}{|c|}{ WLSE } \\
\hline & & & $\begin{array}{l}\overline{\hat{\eta}}_{L} \\
\overline{\widehat{\sigma}}_{L} \\
\widehat{\theta}_{L}\end{array}$ & $\begin{array}{l}\operatorname{MSE}\left(\widehat{\eta}_{L}\right) \\
\operatorname{MSE}\left(\widehat{\sigma}_{L}\right) \\
\operatorname{MSE}\left(\widehat{\theta}_{L}\right)\end{array}$ & $\begin{array}{l}\operatorname{RAB}\left(\hat{\eta}_{L}\right) \\
\operatorname{RAB}\left(\widehat{\sigma}_{L}\right) \\
\operatorname{RAB}\left(\widehat{\theta}_{L}\right)\end{array}$ & $\begin{array}{l}\text { MMSE } \\
\text { MRAB }\end{array}$ & $\begin{array}{l}\overline{\hat{\eta}}_{W} \\
\overline{\widehat{\sigma}}_{W} \\
\widehat{\widehat{\theta}}_{W}\end{array}$ & $\begin{array}{l}\operatorname{MSE}\left(\widehat{\eta}_{W}\right) \\
\operatorname{MSE}\left(\widehat{\sigma}_{W}\right) \\
\operatorname{MSE}\left(\widehat{\theta}_{W}\right)\end{array}$ & $\begin{array}{l}\operatorname{RAB}\left(\widehat{\eta}_{W}\right) \\
\operatorname{RAB}\left(\widehat{\sigma}_{W}\right) \\
\operatorname{RAB}\left(\widehat{\theta}_{W}\right)\end{array}$ & $\begin{array}{l}\text { MMSE } \\
\text { MRAB }\end{array}$ \\
\hline \multirow{27}{*}{100} & \multirow{13}{*}{60} & \multirow{4}{*}{1} & 4.0515 & 1.3811 & 0.2218 & 0.9072 & 4.0952 & 1.0753 & 0.1972 & 0.7242 \\
\hline & & & 3.9961 & 1.1830 & 0.1620 & 0.1931 & 4.0126 & 0.9539 & 0.1533 & 0.1810 \\
\hline & & & 1.5458 & 0.1576 & 0.1955 & & 1.5493 & 0.1433 & 0.1924 & \\
\hline & & & 4.1471 & 1.8059 & 0.2461 & 1.0887 & 4.1839 & 1.4089 & 0.222 & 0.8912 \\
\hline & & \multirow[t]{3}{*}{2} & 4.0761 & 1.3043 & 0.1672 & 0.2027 & 4.1414 & 1.1082 & 0.1614 & 0.1935 \\
\hline & & & 1.5257 & 0.1560 & 0.1947 & & 1.5114 & 0.1564 & 0.1971 & \\
\hline & & & 4.0942 & 1.4625 & 0.2289 & 0.9976 & 4.1236 & 1.1084 & 0.1993 & 0.7193 \\
\hline & & \multirow[t]{3}{*}{3} & 4.0590 & 1.3701 & 0.1735 & 0.2002 & 3.9928 & 0.9058 & 0.1521 & 0.1811 \\
\hline & & & 1.5323 & 0.1601 & 0.1982 & & 1.5562 & 0.1437 & 0.1920 & \\
\hline & & & 4.1208 & 1.6778 & 0.2411 & 1.0733 & 4.1595 & 1.3026 & 0.215 & 0.8431 \\
\hline & & \multirow[t]{3}{*}{4} & 4.0733 & 1.3761 & 0.1722 & 0.2042 & 4.0636 & 1.0716 & 0.1610 & 0.1912 \\
\hline & & & 1.5266 & 0.1660 & 0.1993 & & 1.5375 & 0.1551 & 0.1977 & \\
\hline & & & 4.0775 & 1.2080 & 0.2047 & 0.7785 & 4.0888 & 0.9761 & 0.1851 & 0.6866 \\
\hline & \multirow{11}{*}{80} & \multirow[t]{3}{*}{1} & 4.0369 & 0.9978 & 0.1484 & 0.1764 & 4.1004 & 0.9465 & 0.1530 & 0.1739 \\
\hline & & & 1.5239 & 0.1296 & 0.1760 & & 1.5078 & 0.1373 & 0.1835 & \\
\hline & & & 4.0657 & 1.2917 & 0.2141 & 0.8152 & 4.0865 & 1.0466 & 0.1953 & 0.6878 \\
\hline & & \multirow[t]{3}{*}{2} & 4.0455 & 1.0233 & 0.1498 & 0.1798 & 4.1164 & 0.8923 & 0.1494 & 0.1741 \\
\hline & & & 1.5185 & 0.1307 & 0.1754 & & 1.4952 & 0.1244 & 0.1775 & \\
\hline & & & 4.0845 & 1.2750 & 0.2112 & 0.8451 & 4.095 & 0.9718 & 0.1875 & 0.7049 \\
\hline & & \multirow[t]{3}{*}{3} & 4.0575 & 1.1227 & 0.1579 & 0.1838 & 4.0669 & 1.0079 & 0.1535 & 0.1750 \\
\hline & & & 1.5244 & 0.1376 & 0.1824 & & 1.5266 & 0.1350 & 0.1841 & \\
\hline & & & 4.0828 & 1.2696 & 0.2117 & 0.8306 & 4.1005 & 1.0218 & 0.1916 & 0.7248 \\
\hline & & \multirow[t]{3}{*}{4} & 4.0519 & 1.0819 & 0.1560 & 0.1830 & 4.0914 & 1.0142 & 0.1553 & 0.1775 \\
\hline & & & 1.5246 & 0.1402 & 0.1812 & & 1.5169 & 0.1384 & 0.1855 & \\
\hline & \multirow{3}{*}{100} & & 4.0629 & 1.0169 & 0.1909 & 0.7348 & 4.0905 & 0.8740 & 0.1783 & 0.6133 \\
\hline & & \multirow[t]{2}{*}{-} & 4.0753 & 1.0702 & 0.1473 & 0.1678 & 4.1021 & 0.8487 & 0.1444 & 0.1644 \\
\hline & & & 1.5173 & 0.1173 & 0.1653 & & 1.5078 & 0.1172 & 0.1705 & \\
\hline
\end{tabular}

and the MLEs are better than the MEs via the MMSEs and MRABs, from the numerical results performed via simulation studies.

From Tables 4 and 5, it can be noticed that MSEs, RABs, MMSEs, and MRABs decrease by increasing $m$ (or $n$ ) for fixed values of $n$ (or $m$ ), except for certain unusual cases, and this may be due to data fluctuations.

\section{Concluding Remarks}

We have proposed a new lifetime distribution that could be accomplished by compounding two zero-truncated distributions of Poisson with an ED. It accommodates multiple shapes of the hazard rate function such as decreasing, increasing, and upside-down bathtub shapes. It may represent the failure time of a system connected in the form of a parallel-series structure. Some properties of the EDPD have been studied, including its PDF, shapes of the HRF, $r$-th moments, PDF of the $i$-th order statistic, mean, variance, Bonferroni curve, Lorenz curve, Rényi entropy, and Shannon's entropy. Two real datasets are considered to compare the EDPD, EWPD, EEPD, EPD, PED, and ED. The comparison reveals that the EDPD is better than the other five distributions to fit the data considered. We have addressed four estimation methods to estimate the involved parameters on the basis of progressive type II censoring. The methods are estimations of ML, M, LS, and WLS. A simulation analysis was conducted to determine the best performing estimators, and numerical computations were carried out based on four different progressive CSs.

Among the features and motivations of the EDPD are as follows:

(1) The PDF and HRF of the EDPD have closed forms.

(2) The different shapes of the HRF of EDPD make it more appropriate to fit multiple real data.

(3) It may be used to describe the lifetime of a system connected as a parallel-series structure.

(4) It is better than some other distributions such as the EWPD, EEPD, EPD, PED, and ED to suit multiple real data.

\section{Appendix}

The likelihood equations for $\eta, \sigma$, and $\theta$ are given by 


$$
\begin{aligned}
& 0=\frac{\partial E}{\partial \eta}=\frac{m}{\eta}-\frac{n}{1-e^{-\eta}}+\sum_{i=1}^{m}\left(\Delta\left(t_{i}\right)+R_{i} \frac{e^{\eta}-\Delta\left(t_{i}\right) \exp \left[\eta \Delta\left(t_{i}\right)\right]}{e^{\eta}-\exp \left[\eta \Delta\left(t_{i}\right)\right]}\right) \\
& 0=\frac{\partial E}{\partial \sigma}=\frac{m}{\sigma}-\frac{m}{1-e^{-\sigma}}+\sum_{i=1}^{m}\left(e^{-\theta t_{i}}+\left[\frac{\eta}{e^{-\sigma}-1}\left(\exp \left[-\sigma-\theta t_{i}+\sigma e^{-\theta t_{i}}\right]+\Delta\left(t_{i}\right)-1\right) \times\left(1-\frac{R_{i}}{\exp \left[\eta\left(1-\Delta\left(t_{i}\right)\right)\right]-1}\right)\right]\right) \\
& 0=\frac{\partial E}{\partial \theta}=\frac{m}{\theta}-\sum_{i=1}^{m}\left(t_{i}+\sigma t_{i} e^{-\theta t_{i}}+\left[\frac{\eta \sigma}{1-e^{\sigma}} t_{i} \exp \left[-\theta t_{i}+\sigma e^{-\theta t_{i}}\right]\left(1-\frac{R_{i}}{\exp \left[\eta\left(1-\Delta\left(t_{i}\right)\right)\right]-1}\right)\right]\right)
\end{aligned}
$$

\section{Data Availability}

The data used to support the findings of this study are included within the article.

\section{Conflicts of Interest}

The authors declare that they have no conflicts of interest regarding the publication of this paper.

\section{Authors' Contributions}

AFH contributed to the idea and theoretical results, as well as writing and reviewing the paper. SAA contributed to the numerical results and the real example application.

\section{Acknowledgments}

The authors would like to thank both Professor Alaa H. Abdel-Hamid (Beni-Suef University, Egypt) for reviewing this paper and Professor Coşkun Kuş (Selçuk University, Turkey) for his comments that improved the paper.

\section{References}

[1] C. Kuş, "A new lifetime distribution," Computational Statistics \& Data Analysis, vol. 51, pp. 4497-4509, 2007.

[2] V. G. Cancho, F. Louzada-Neto, and G. D. C. Barriga, "The Poisson-exponential lifetime distribution," Computational Statistics \& Data Analysis, vol. 55, no. 1, pp. 677-686, 2011.

[3] M. M. Ristić and S. Nadarajah, "A new lifetime distribution," Journal of Statistical Computation and Simulation, vol. 84, pp. 135-150, 2014.

[4] F. Louzada, M. Roman, and V. G. Cancho, "The complementary exponential geometric distribution: model, properties, and a comparison with its counterpart," Computational Statistics \& Data Analysis, vol. 55, no. 8, pp. 2516-2524, 2011.

[5] F. Louzada, V. Marchi, and J. Carpenter, "The complementary exponentiated exponential geometric lifetime distribution," Journal of Probability and Statistics, vol. 2013, Article ID 502159, 12 pages, 2013.

[6] E. Mahmoudi and A. Sepahdar, "Exponentiated WeibullPoisson distribution: model, properties and applications," Mathematics and Computers in Simulation, vol. 92, pp. 76-97, 2013.

[7] A. H. Abdel-Hamid and A. F. Hashem, "A new lifetime distribution for a series-parallel system: properties, applications and estimations under progressive type-II censoring," Journal of Statistical Computation and Simulation, vol. 87, no. 5, pp. 993-1024, 2017.
[8] A. H. Abdel-Hamid and A. F. Hashem, "A new compound distribution based on a mixture of distributions and a mixed system," Comptes Rendus de L'Academie Bulgare des Sciences, vol. 71, no. 11, pp. 1439-1450, 2018.

[9] S. Nadarajah, A. H. Abdel-Hamid, and A. F. Hashem, "Inference for a geometric-Poisson-Rayleigh distribution under progressive-stress accelerated life tests based on type-I progressive hybrid censoring with binomial removals," Quality and Reliability Engineering International, vol. 34, no. 4, pp. 649-680, 2018.

[10] R. E. Glaser, "Bathtub and related failure rate characterizations," Journal of the American Statistical Association, vol. 75, no. 371, pp. 667-672, 1980.

[11] B. C. Arnold, N. Balakrishnan, and H. N. Nagaraja, A First Course in Order Statistics, John Wiley \& Sons, New York, NY, USA, 1992.

[12] H. A. David and H. N. Nagaraja, Order Statistics, John Wiley \& Sons, Hoboken, NJ, USA, 2003.

[13] C. Canuto, M. Y. Hussaini, A. Quarteroni, and T. A. Zang, Spectral Methods: Fundamentals in Single Domains, SpringerVerlag, New York, NY, USA, 2006.

[14] C. E. Shannon, "Prediction and entropy of printed English," Bell System Technical Journal, vol. 30, no. 1, pp. 50-64, 1951.

[15] A. Rényi, On Measures of Entropy and Information: Proceedings of the 4th Berkeley Symposium on Mathematical Statistics and Probability, pp. 547-561, University of California Press, Berkeley, CA, USA, 1961.

[16] N. R. Mann, R. E. Schafer, and N. D. Singpurwalla, Methods for Statistical Analysis of Reliability and Life Data, Wiley, New York, NY, USA, 1974.

[17] W. Q. Meeker and L. A. Escobar, Statistical Methods for Reliability Data, Wiley, New York, NY, USA, 1998.

[18] J. F. Lawless, Statistical Models and Methods for Lifetime Data, Wiley, New York, NY, USA, 2-nd edition, 2003.

[19] N. Balakrishnan and R. Aggarwala, Progressive Censoring: Theory, Methods, and Applications, Birkhäuser, Boston, MA, USA, 2000.

[20] N. Balakrishnan, A. Childs, and B. Chandrasekar, "An efficient computational method for moments of order statistics under progressive censoring," Statistics \& Probability Letters, vol. 60, no. 4, pp. 359-365, 2002.

[21] J. J. Swain, S. Venkatraman, and J. R. Wilson, "Least-squares estimation of distribution functions in johnson's translation system," Journal of Statistical Computation and Simulation, vol. 29, no. 4, pp. 271-297, 1988.

[22] R. Aggarwala and N. Balakrishnan, "Some properties of progressive censored order statistics from arbitrary and uniform distributions with applications to inference and simulation," Journal of Statistical Planning and Inference, vol. 70, no. 1, pp. 35-49, 1998. 
[23] M. T. Madi and M. Z. Raqab, "Bayesian prediction of rainfall records using the generalized exponential distribution," Environmetrics, vol. 18, no. 5, pp. 541-549, 2007.

[24] M. Z. Raqab, A. Asgharzadeh, and R. Valiollahi, "Prediction for Pareto distribution based on progressively type-II censored samples," Computational Statistics \& Data Analysis, vol. 54, no. 7, pp. 1732-1743, 2010.

[25] R. L. Smith and J. C. Naylor, "A comparison of maximum likelihood and bayesian estimators for the three- parameter Weibull distribution," Applied Statistics, vol. 36, no. 3, pp. 358-369, 1987.

[26] N. Balakrishnan and R. A. Sandhu, "A simple simulational algorithm for generating progressive type-II censored samples," The American Statistician, vol. 49, no. 2, pp. 229-230, 1995. 